\title{
A STUDY ON THE STATISTICAL CALIBRATION OF THE HOLTROP AND MENNEN APPROXIMATE POWER METHOD FOR FULL HULLFORM, LOW FROUDE NUMBER VESSELS
}

\author{
Lampros Nikolopoulos* \\ Department of Naval Architecture, Ocean and Marine Engineering \\ University of Strathclyde \\ Email :lampros.nikolopoulos@strath.ac.uk
}

The paper herein presents a statistical calibration study of the approximate power method of Holtrop and Mennen focused on adapting the latter to vessels characterized by "full" hull forms and low design and operating speeds and thus low Froude numbers. The fitting of the method is done by adjusting the constants, coefficients and components of the method's equations by a systematic variation process controlled by genetic algorithms. The database that the method is calibrated against is consisted by model test results from modern (built between 2010 and 2016) bulk carriers and tankers, the KVLCC2 and follows a multi-staged approach, calibrating first the model for the prediction of the total resistance and applying the self-propulsion equations afterwards. The uncertainty of the new improved method is assessed and modeled with a non-linear regression equation in order to enable the use of the calibrated method in the early ship design and optimization process. 
2 One of the most important aspects of the design and study of vessels and ocean structures is the prediction of the resistance and thus powering requirements for a range of operational speeds. Given the constant societal and legislative pressure for the reduction of the carbon footprint of shipping and maritime activities, the accurate and reliable powering prediction from an early stage and tightly integrated within the process itself as the respective optimization studies is imperative. While towing tank model testing has been since early times the most reliable way for power predictions, due to its high cost and demand for a fixed and given geometry can be used only in the stage of basic design when the design parameters related to the vessel's hull geometry are fixed and serves as a final validation and benchmark to be used afterwards in the conclusion of the shipbuilding process during sea trials. On the other hand, the last decade has seen the exponential growth of the application of Computational Fluid Dynamic solvers that solve the Reynolds Averaged Navier Stokes (RANS) equations over the hull form in finite volume approaches [1], [2]. While originally the computational cost was penalizing its application in early design stages, the advances in computing hardware and software allowed the integration of CFD in the early ship hull form design and optimization [1]. Such methods however can be employed in applications where variables such as the principal particulars have been fixed or have a small variance with the focus being on the variation of local geometry and topology characteristics that have a significant effect on local flow phenomena [1]. However, when the application in question is a global optimization study, where there is a large variance on principal particulars (vessel's dimensions), structural and cargo arrangements, there is an apparent need for a large number of optimization variants and thus evaluations (usually in the order of thousands). The application of CFD in such cases is impractical as the optimization algorithm will eventually require hundreds of thousands computational hours even in high performance computers, and empirical or statistical methods better suited. The most prominent of these is the Approximate Power Prediction Method by Holtrop and Mennen [3] together with its revision [4]. Although this methodology provides sufficient accuracy, the statistical sample of the hull forms on which it is based dates back in the 1970s and 1980s. Such hulls, although roughly similar, have some distinct deviations from modern commercial vessels. In the paper presented herein, the authors attempt to make a calibration of the Holtrop and Mennen methodology via a systematic variation with the use of genetic algorithms. The calibration is based on a statistical sample of model test results of low Froude number $(\mathrm{Fn}$ ) full hull forms (with $\mathrm{Cb}$ greater than 0.7 ) of modern commercial bulk carriers and tankers and its focus is on the integration of the new coefficients in a holistic methodology for the optimization of large bulk carriers. The uncertainty of the new coefficients is also taken into account based on the sea trial results of an expanded statistical sample.

\section{General}

The Holtrop and Mennen method [3], [4] is currently considered as one of the most accurate and efficient methods for the estimation of the resistance and propulsion power requirements of conventional mono-hull vessels at the initial stages of the design. It is an empirical method consisting of equations for the various resistance components that derive from the statistical analysis and regression of a database with a large number of model test results. It was developed in the early 1970s by Jan Holtrop and Frits Mennen when they were working in the MARIN research centre and towing tank. They focused on developing a modern way to carry out data analysis with a focus on the extrapolation of model tests. Initially, the method was designed for internal purposes so MARIN could make more accurate predictions. The two started with the system analysis and then reanalysed the model tests and the full-scale trials. The target was to have a component-wise prediction method that would show the difference between model and full-scale to serve the extrapolation of the model experiments [5].

The resistance decomposition as well as hull form considerations taken and the large number of input variables make the Holtrop Methodology an ideal candidate for an initial early stage too (IMO Level 1 Stage) for predicting the powering requirements of a given hull. For applications where the range of design parameters is large ${ }^{1}$ and subject to change, the methodology can provide good accuracy, correct trends and variant ranking in terms of resistance. However, for reasons of clarity the following restrictions should be noted:

1. The Holtrop methodology is based on the regression analysis of 334 ship models but within a range of dimension ratios (L/B, B/T, T/L, Cb, Cp, Cwp etc) [4]. Although the range of applicability is significant and all typical vessel ratios are covered sufficiently, in cases of new designs where their ratios are close to the margins of the ones studied by Holtrop, a drop in accuracy must be taken into account.

2. For non-conventional hull forms and all vessels having hull geometries significantly different from the original database used the accuracy of subject methodology is limited. Additionally, for vessels fitted with hydrodynamic improvement energy devices (commonly referred to as Energy Saving Devices) that significantly affect various flow phenomena at the stern of such vessels the prediction error is increased as such cases are not included in the original database used by Holtrop and Mennen.

\footnotetext{
${ }^{1}$ Such as in the concept and preliminary ship design stages where the principal particulars of the vessel are not fixed.
} 
Following the development of the methodology and a second publication from Hotrop and Mennen [4] on its updated coefficients, its use is very widespread both in a plethora of initial design applications but also in ship operation and performance simulation modules with some recent examples in [6], [7], [8], [9]. Interestingly though, despite the above and the development of other statistical based methods for the problem of ship propulsion power prediction, the literature available at the time of writing lacks studies on the systematic calibration of the Holtrop and Mennen methodology based on statistical samples from either model tests, sea trials or CFD results.

The study presented herein aims to assess the deviation of the Holtrop and Mennen prediction when compared to model test results, in Section 2, for a database of full hull form and low Froude number modern existing vessels. Afterwards, in Section 3 the various constants and coefficients used in Holtrop and Mennen methodology are adjusted through a multi-staged approach for both the bare hull resistance as well as the propulsion power prediction. The error of the new methodology is modelled with non-linear regression formula and presented in Section 4 and lastly the results and future work are discussed in Section 5.

\section{Vessel Model Test Database}

In order to assess the level of accuracy of the methodology in question, a database was built comprised from model test results of different vessels. The vessels collected are existing or vessels under construction of full hull form that represent modern ship design trends. Their principal characteristics which were used as input for the Holtrop powering prediction are shown on the table below:

\begin{tabular}{|l|c|c|c|c|c|c|c|}
\hline Principal Particular & VSL01 & VSL02 & VSL03 & VSL04 & VSL05 & VSL06 & VSL07 \\
\hline Vessel Type & KVLCC2 & VLCC & Newcastlemax & Capesize & Capesize & Ultramax & Ultramax \\
\hline Lwl (m) & 335 & 322 & 298.61 & 291 & 292 & 198 & 200 \\
\hline Lbp (m) & 334 & 328 & 294 & 286 & 288 & 195 & 195 \\
\hline B (m) & 61 & 60 & 50 & 45 & 45 & 32.26 & 32 \\
\hline Draft (m) & 20.8 & 21.6 & 18.5 & 18.3 & 18.15 & 12.9 & 11.3 \\
\hline Displacement (m) & 343176.4 & 333410.3 & & & 202174.2 & 68864 & 61000 \\
\hline Cb & 0.8098 & 0.7989 & 0.837 & 0.845 & 0.8595 & 0.8486 & 0.86 \\
\hline LCB (\%) & 0.035 fwd & 0.03188 & 0.02368 & 0.0175 & -0.0162 & -0.0159 & 0.0285 \\
\hline Bulbous Bow transverse Area (m2) & 100 & 123.9 & 100 & 100 & 0 & 10 & 63.053 \\
\hline Centre of bulbous bow transverse area (m) & 7.5 & 11 & 7.5 & 7.5 & 6 & 5 & 6 \\
\hline Cm & 0.998 & 0.998 & 0.998 & 0.997 & 0.9981 & 0.9981 & 0.9953 \\
\hline Transom Transverse Area (m) & 30 & 30 & 30 & 30 & 30 & 30 & 30 \\
\hline Cstern & 0 & 0 & 0 & 0 & 0 & 0 & 0 \\
\hline Wetted Surface (m2) & 29629.27 & 28226.2 & & & 20959.7 & 10196.8 & 9706 \\
\hline Cp & 0.8114 & 0.8005 & 0.8538 & 0.8538 & 0.8538 & 0.8538 & 0.864 \\
\hline
\end{tabular}

Table [1]: Particulars of vessels examined

From table [1] we can observe that the vessel model test database that serves as the calibration basis is made of full hull forms of bulk carriers and tankers including also vessels that were able to transit the old Panama Canal (PANAMAX beam dimension) resulting into adjustments of their length to beam ratio.

In the figures [1] through [11] the percentage of difference between the measured EHP during the model tests of the above vessel cases and the predicted EHP according to Holtrop's method is depicted. This difference is calculated over the entire range of speeds available in the model tests in order to have a larger number of points (speed, power) that will be used for the calibration studies, as well as assess the effect of speed on the prediction accuracy. 

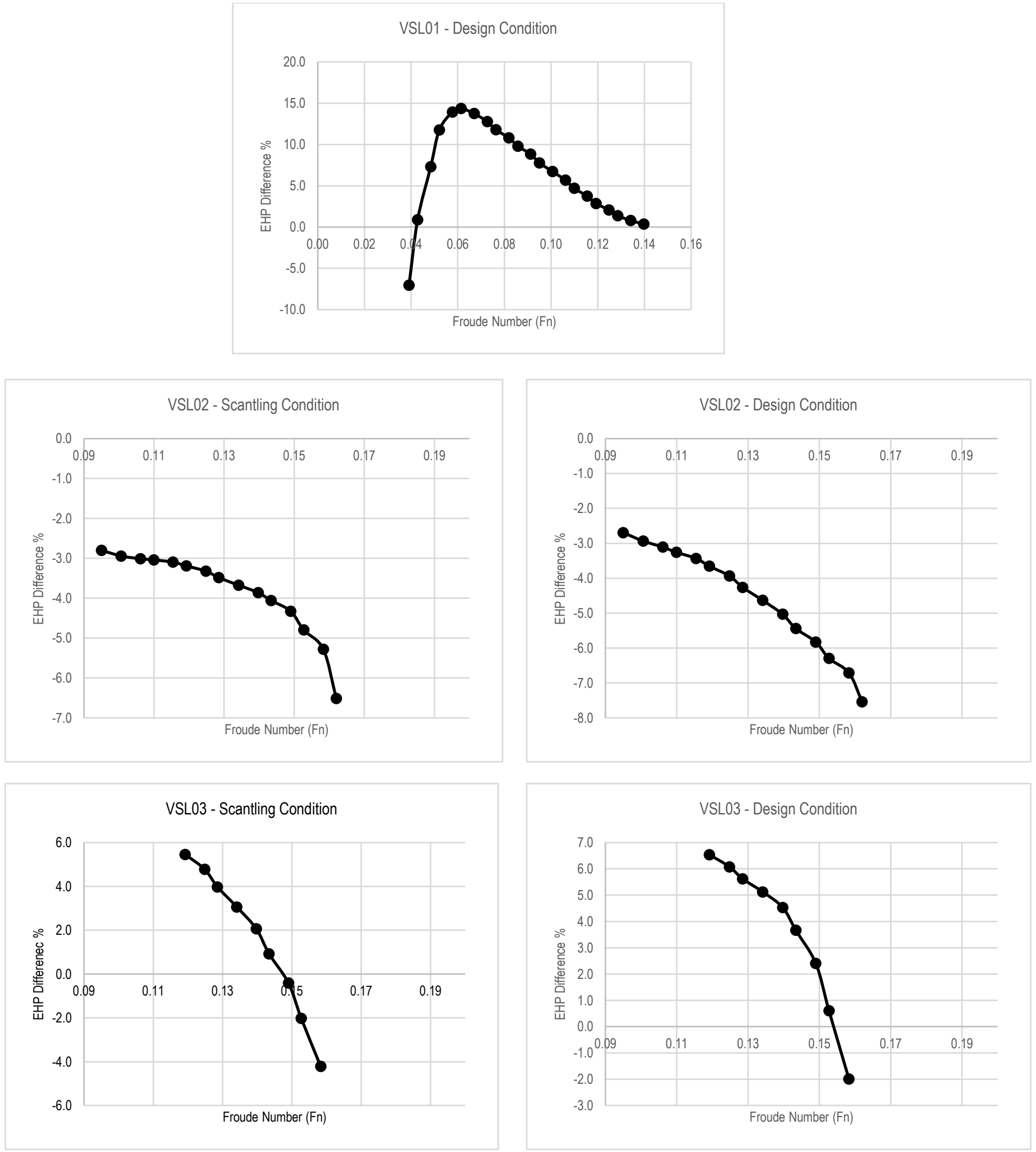

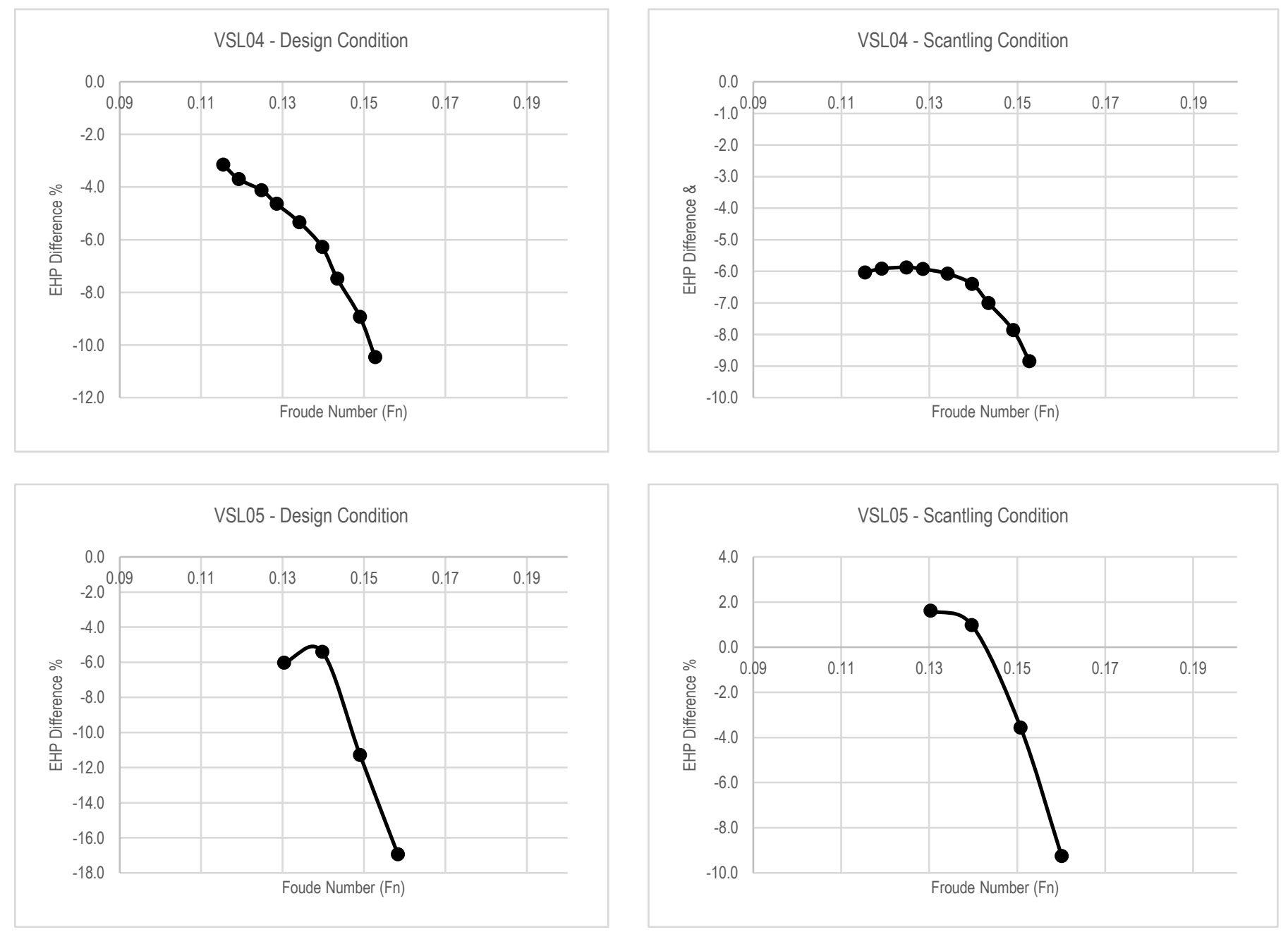

1
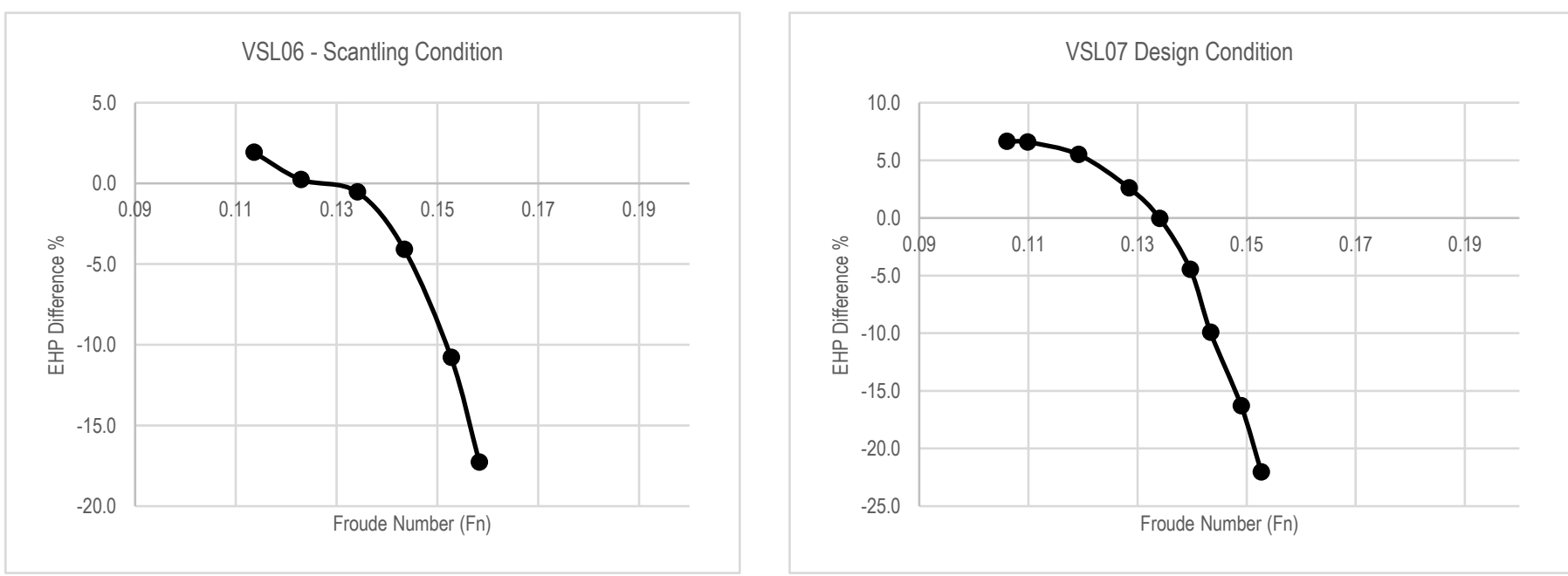

2

Figures [1] to [11] : Distribution of the EHP difference (\%) over different speeds - LADEN Condition 

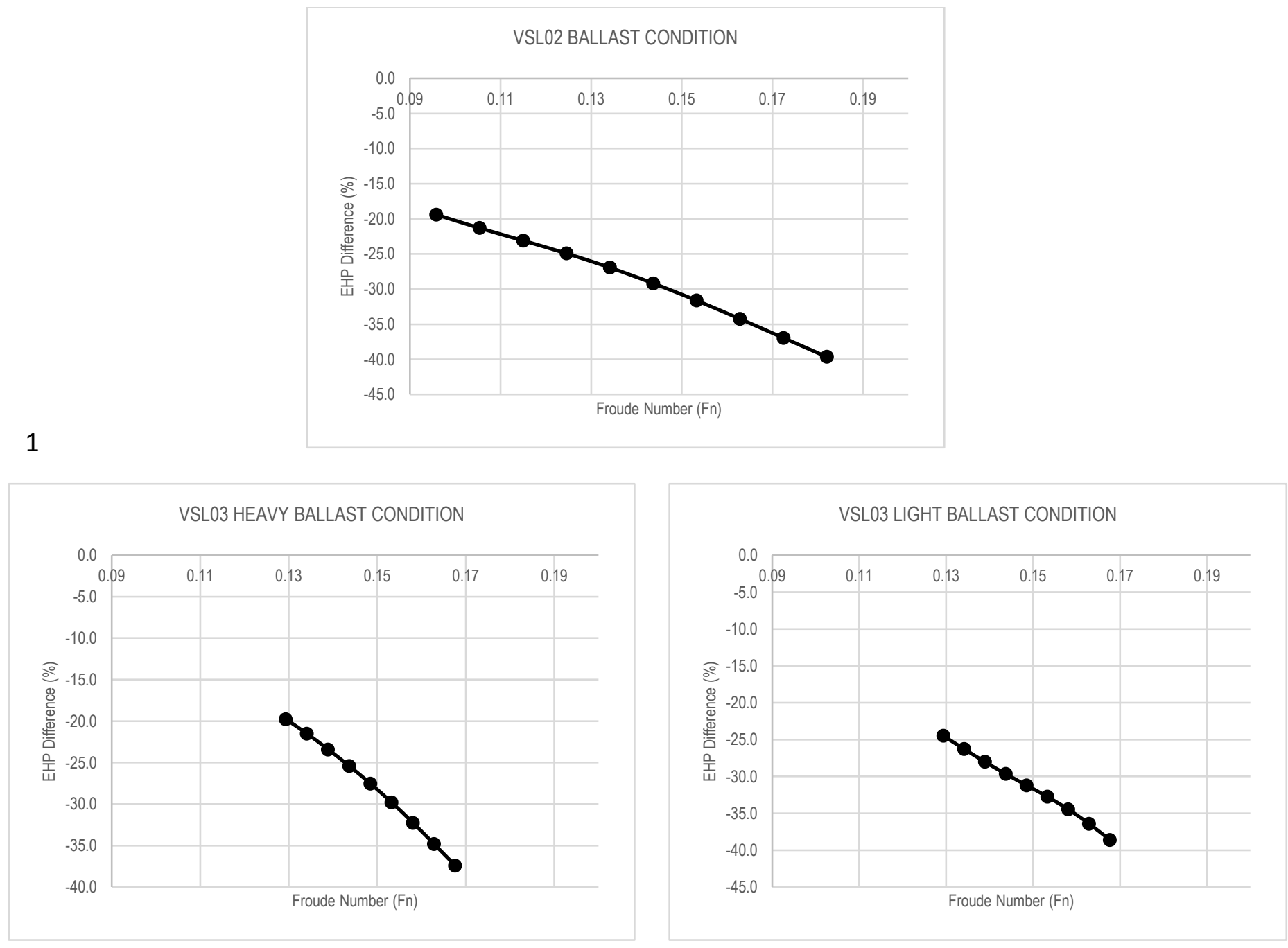

2
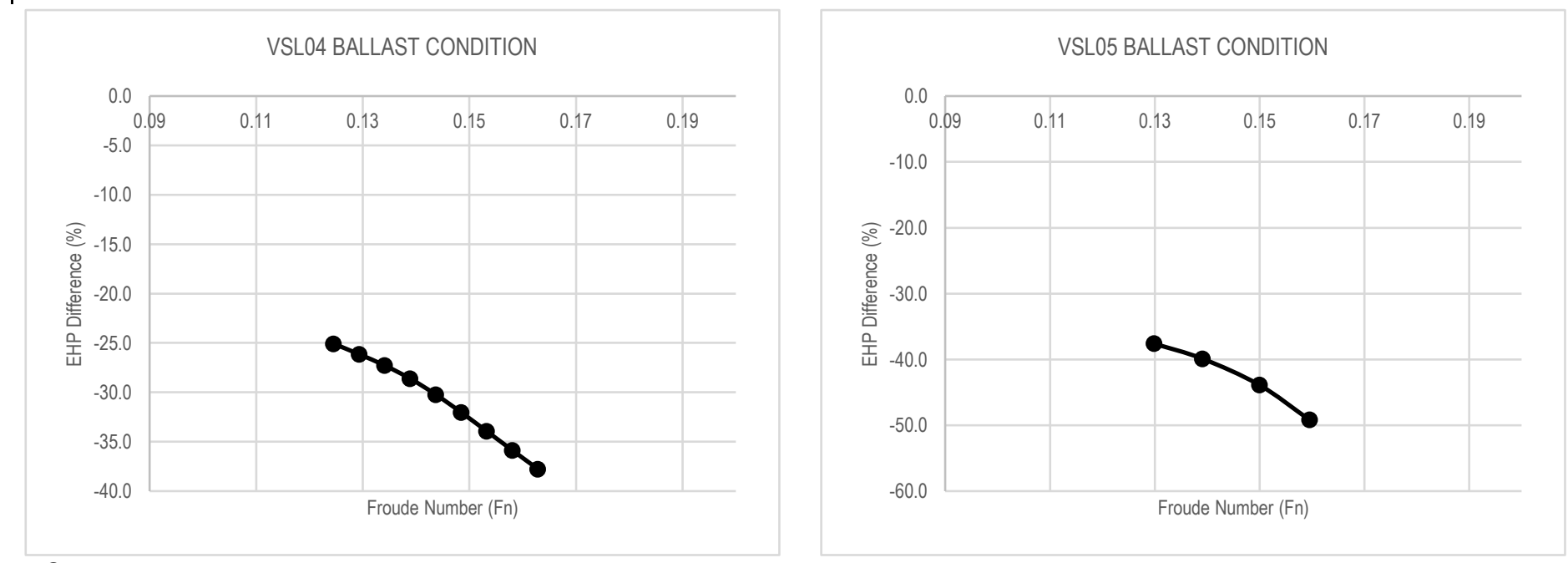

3

4

15

6 

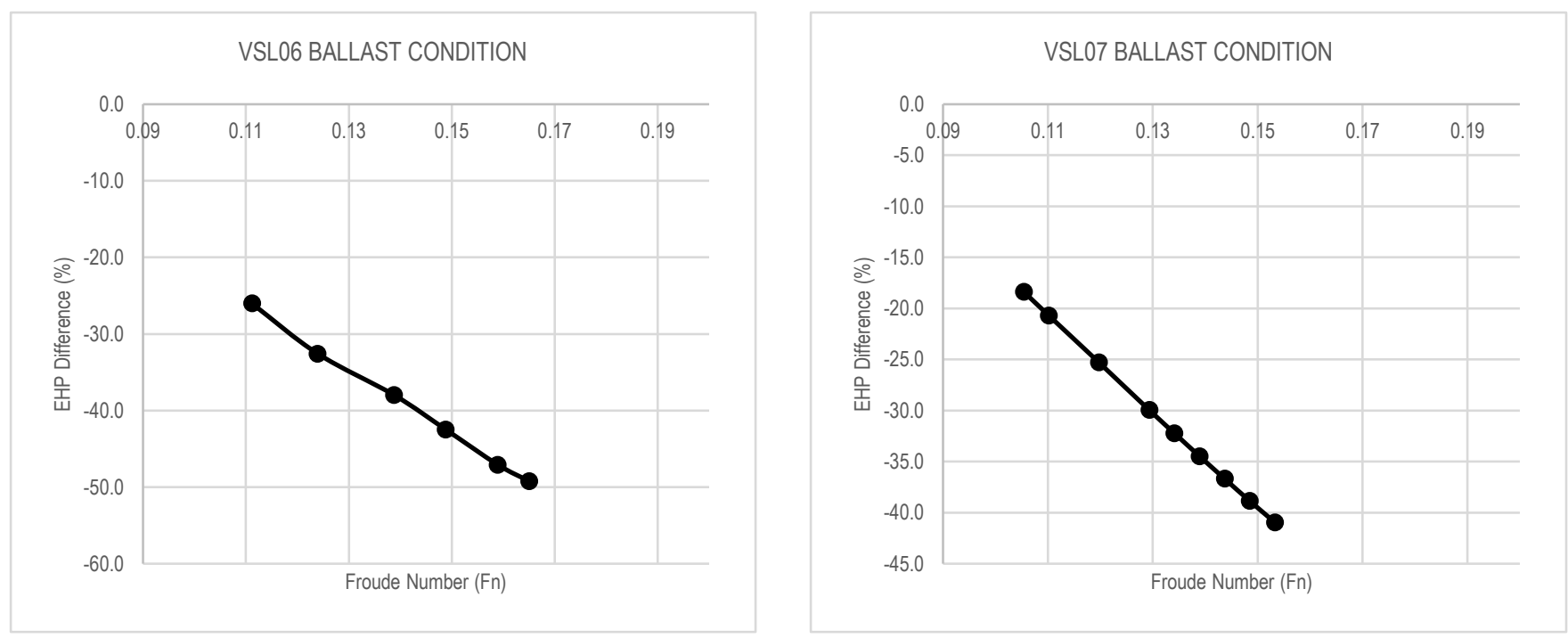

Figures [11] to [17] : Distribution of the EHP difference (\%) over different speeds - BALLAST Condition

For the proper interpretation of the above figures the assumption that a positive $(+)$ difference percentage corresponds to an overestimation of the resistance by the Holtrop and Mennen method whereas a negative (-) difference corresponds to a respective underestimation. From the results the following can be noted:

\section{For the Design and Scantling Conditions}

A very high sensitivity and nearly linear correlation to the operating speed is evident resulting to an increased underestimation of the resistance by increasing Froude number.

1. For 5 out of 11 cases (45.5\%) Holtrop and Mennen underestimate the resistance for the entire speed range ranging from $1 \%$ to $16 \%$.

2. For the other 5 cases, in low Froude numbers Holtrop and Mennen overestimate the resistance by maximum $10 \%$ in the lowest respective speeds but by increasing Fn the relative overestimation is linearly decreasing up to a Froude number in the range of 0.12 to 0.17 (depending on each vessel case) where there is a transition to underestimation of the resistance by Holtrop and Mennen, while in even higher speeds this can be up to maximum $5 \%$. The trend of the linear increase of the underestimation percentage by increasing speed is still very strong and evident as in the previous cases.

3. For the KVLCC2 Case: The Vessel 01 graph corresponding to the distribution of the EHP difference of the KVLCC2 vessel between the Holtrop and Mennen prediction and its model test results is very interesting to comment. At the very low Froude numbers of approximately 0.002 the Holtrop and Mennen method is underestimating the resistance while at 0.04 it marginally overestimates it. The overestimation percentage is increasing by increasing Froude number up to a maximum of $15 \%$ at 0.055 . From this Froude number, the overestimation percentage is decreasing up to 0.13 where it is practically zero. When compared to the other vessel cases of the herein study, this peculiar behaviour at low speeds up to 0.06 Froude number, according to the Authors' understanding, can be attributed to the overestimation of various resistance components such as the influence of the bulbous bow, the viscous pressure and the wave resistance. Unfortunately, the other vessel cases studied herein did not include tests at such low Froude numbers during their model testing, in order to see the behaviour of different hulls and geometries at low Froude numbers.

\section{Ballast Condition}

In contrast to the Laden conditions, in the ballast condition for all vessels, a significant underestimation of a higher relative magnitude is evident. Similarly, to the laden condition, the underestimation percentage is increasing almost linearly by the increase of the Froude number from about $20 \%$ at the lower range up to about $50 \%$ for the high range. The only nonlinear increase is for the case of VSL04, VSL05 and at High Froude numbers of VSL03 where a slight parabola is observed. The steepness of the curve is vessel dependent but for all cases similarities can be observed.

Similarly, to the Effective Horsepower (and thus resistance) difference depicted above, from Figure [17] to [27] and [28] to [34] one can see the distribution of the error between the Holtrop and Mennen methodology and model test prediction for the Shaft Horsepower (SHP) in the laden and ballast conditions respectively. 

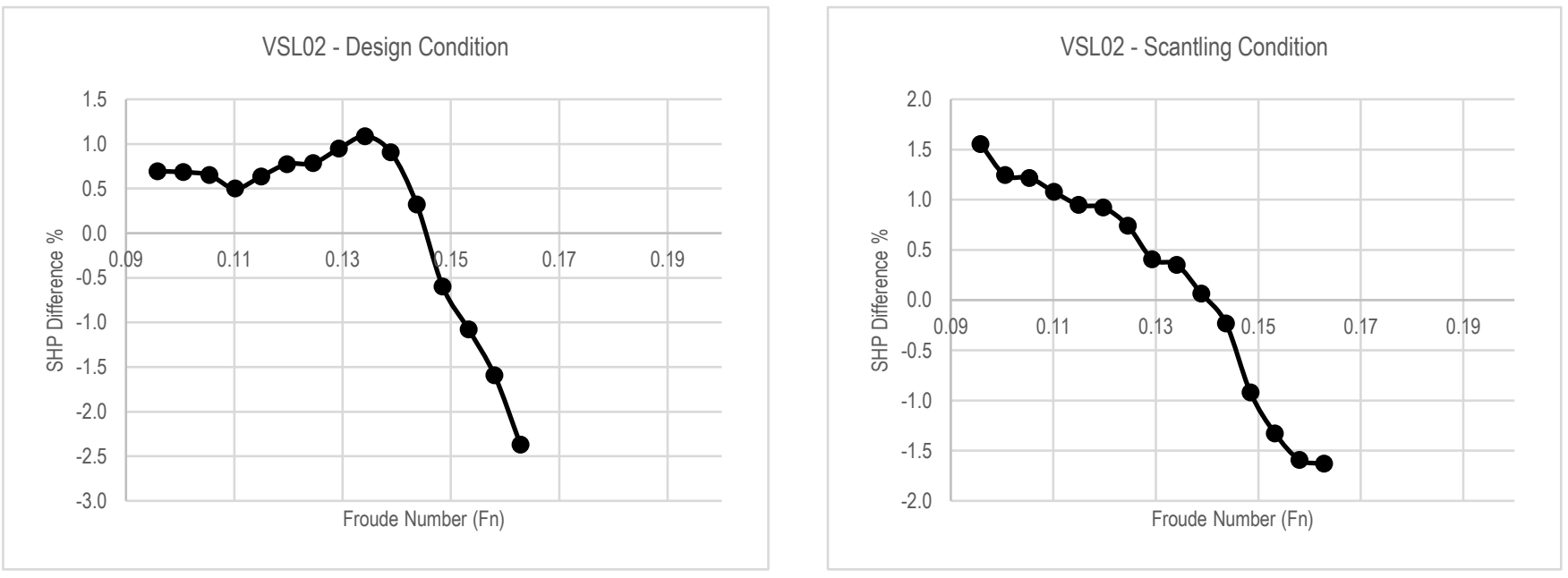

2
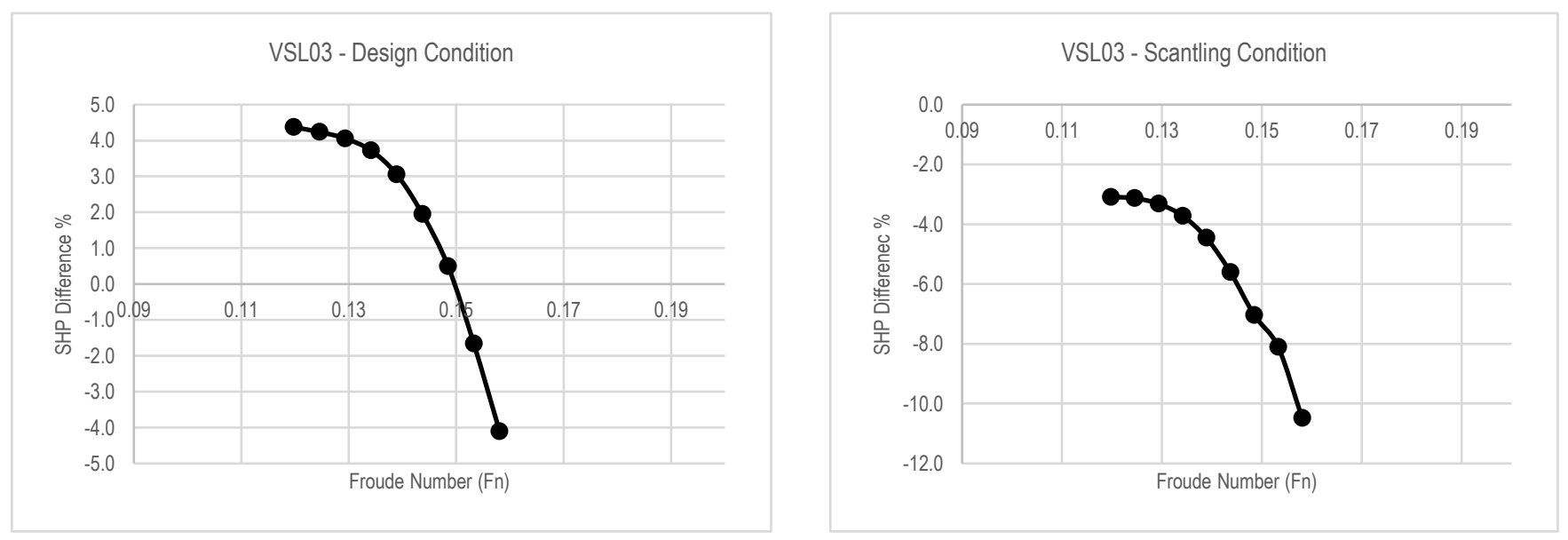

3
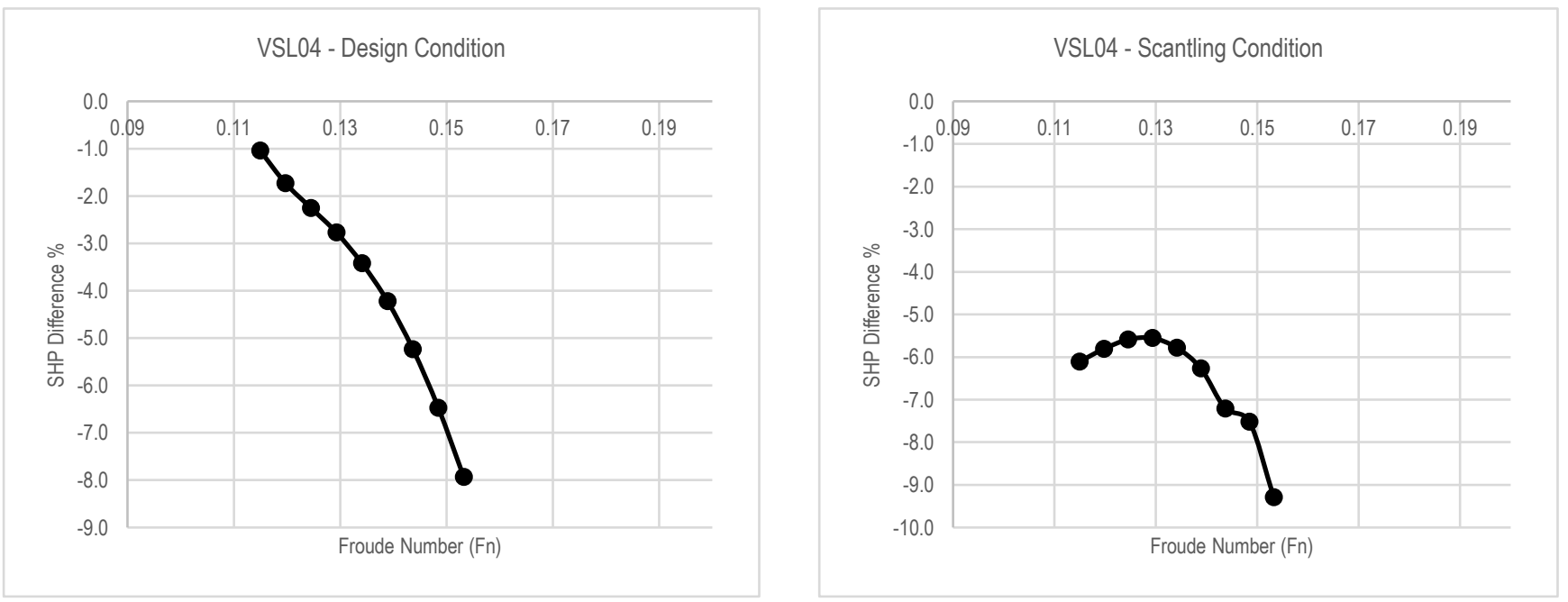

4

5

6 

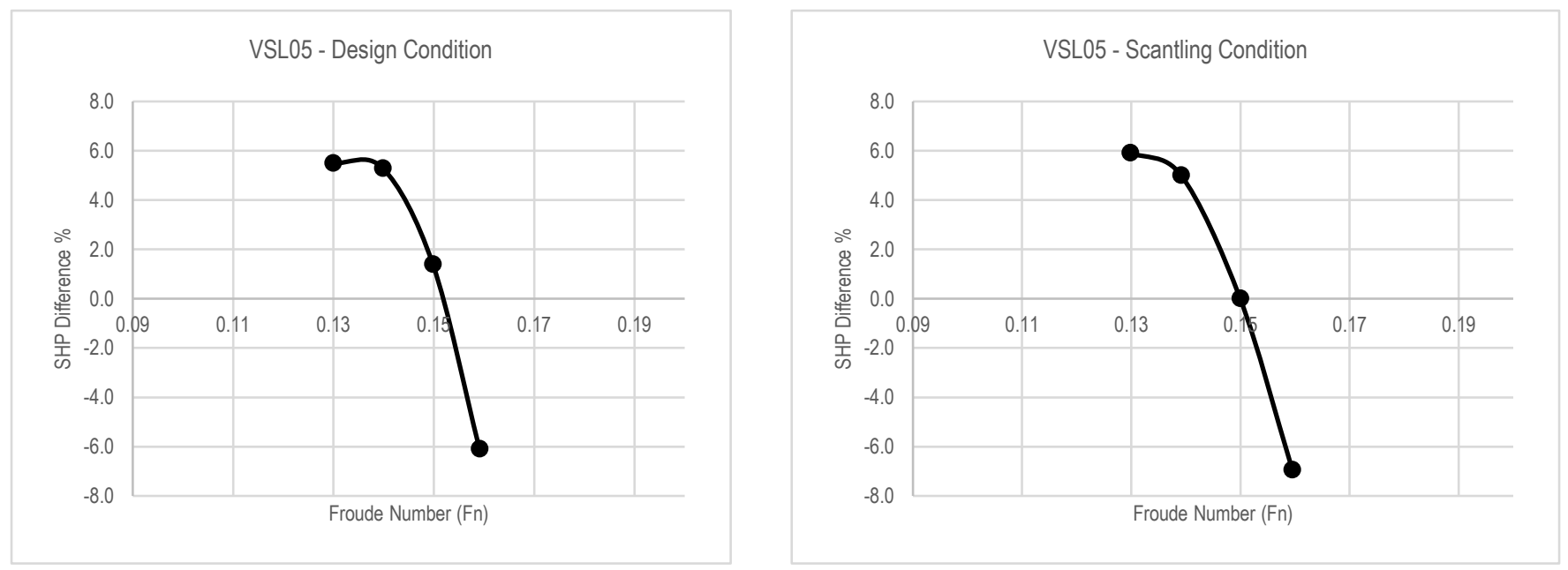

1
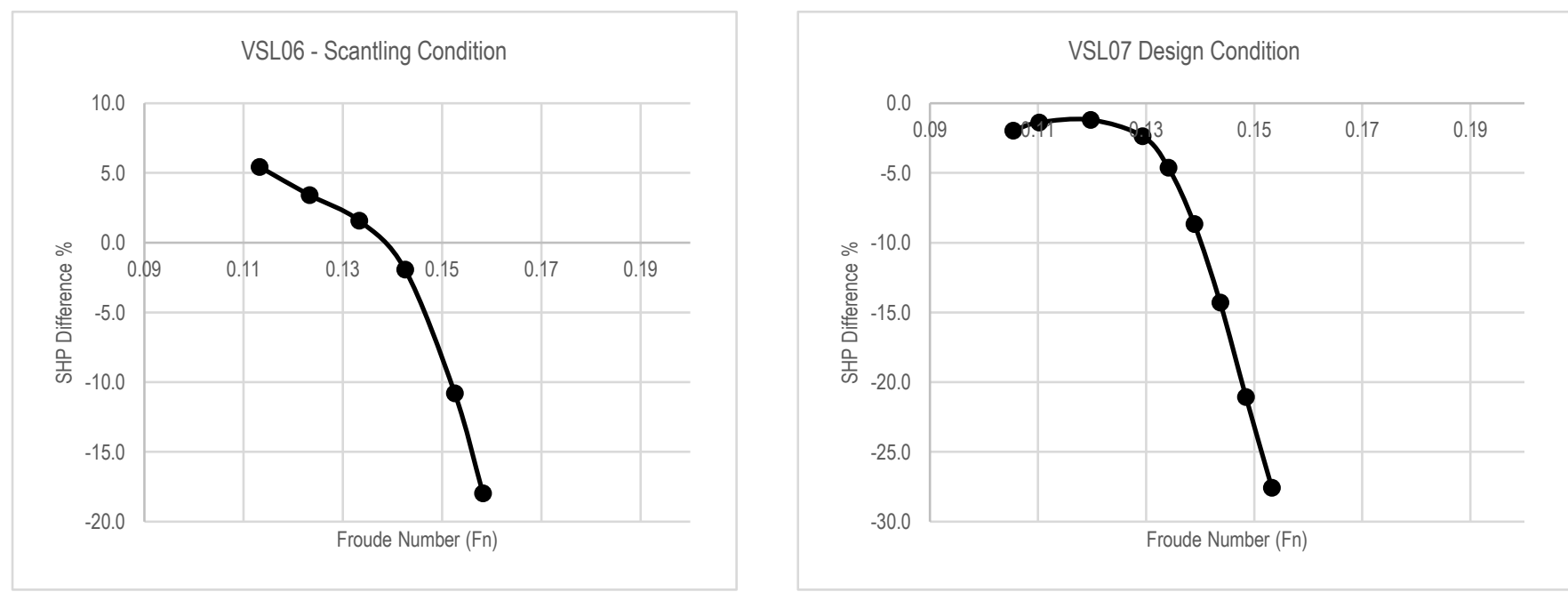

2

3

4

5

Figures [17] to [27]: Shaft Power prediction error (\%) distribution over different speeds - LADEN Condition 

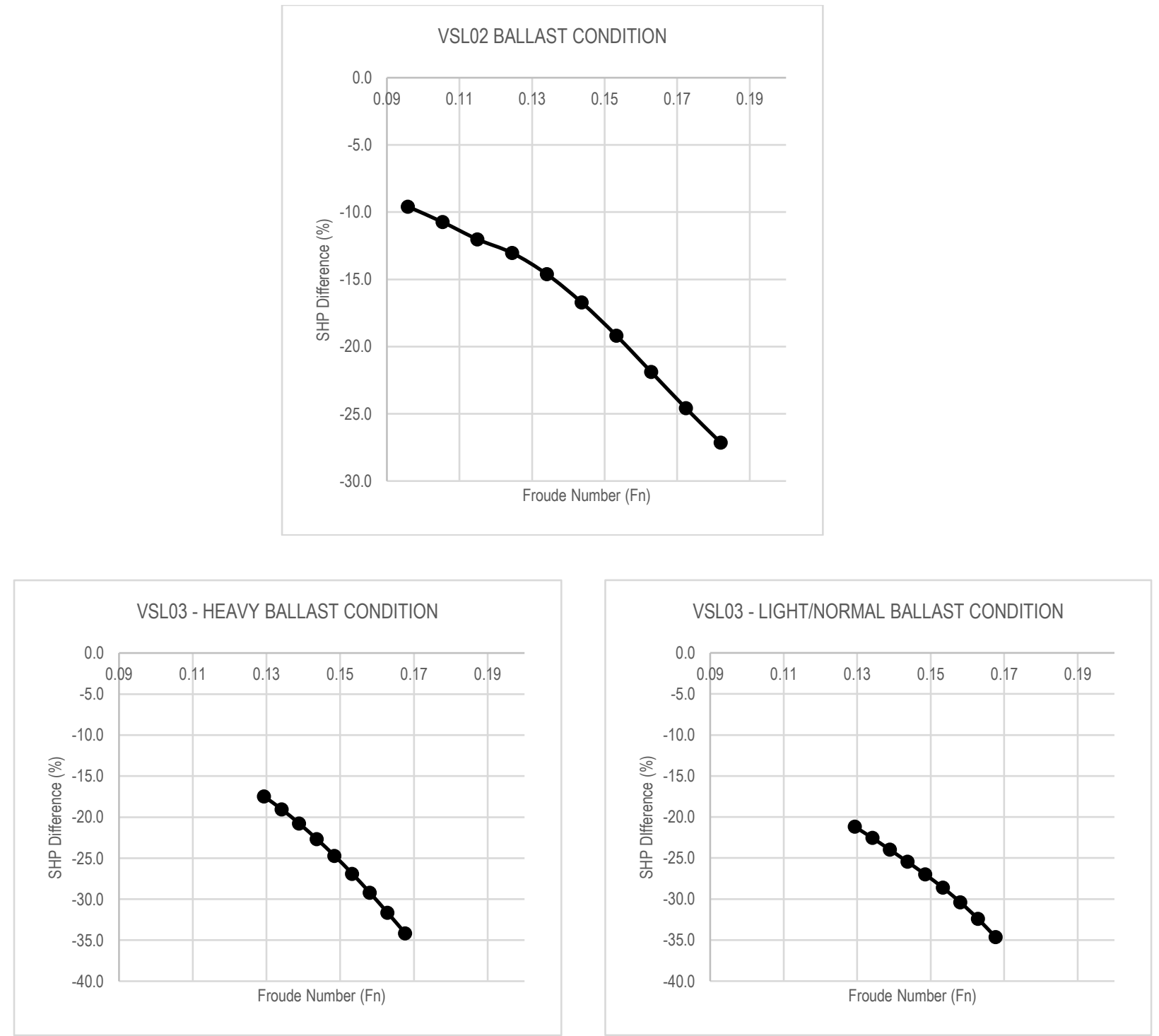

3
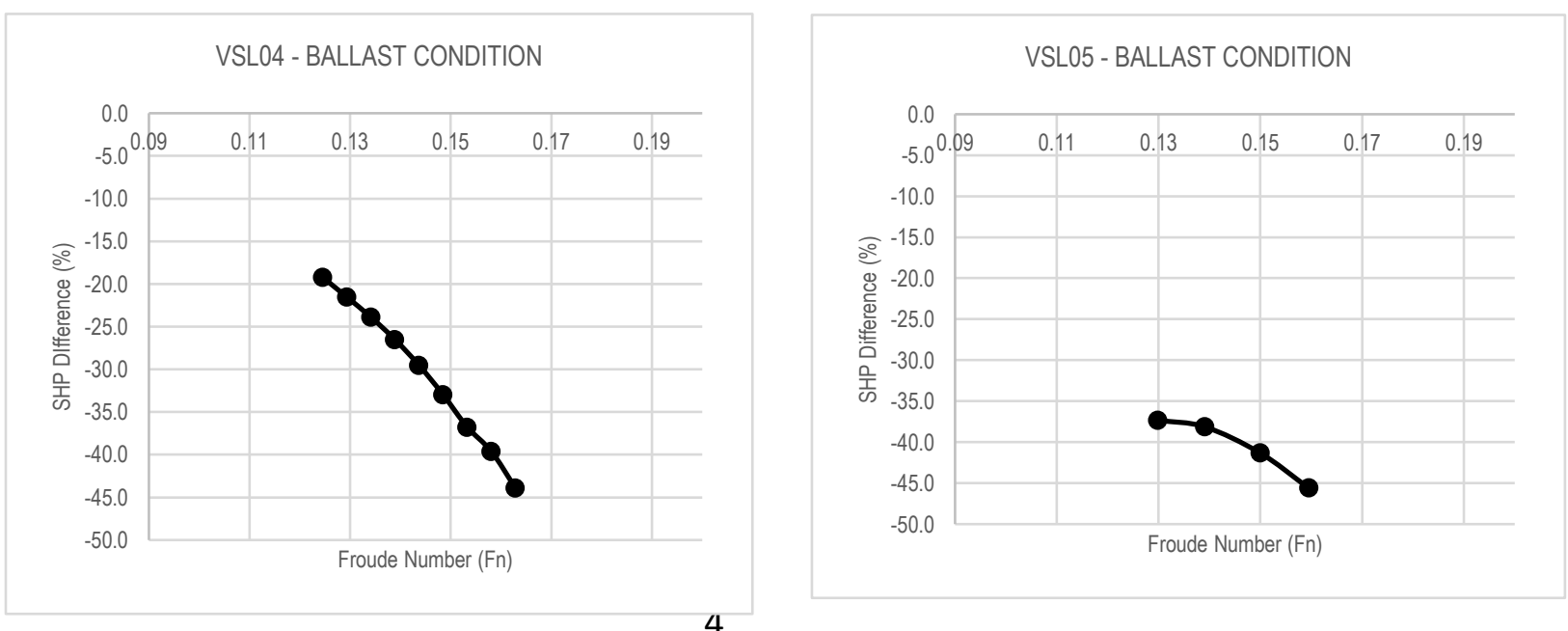

5

6
7 

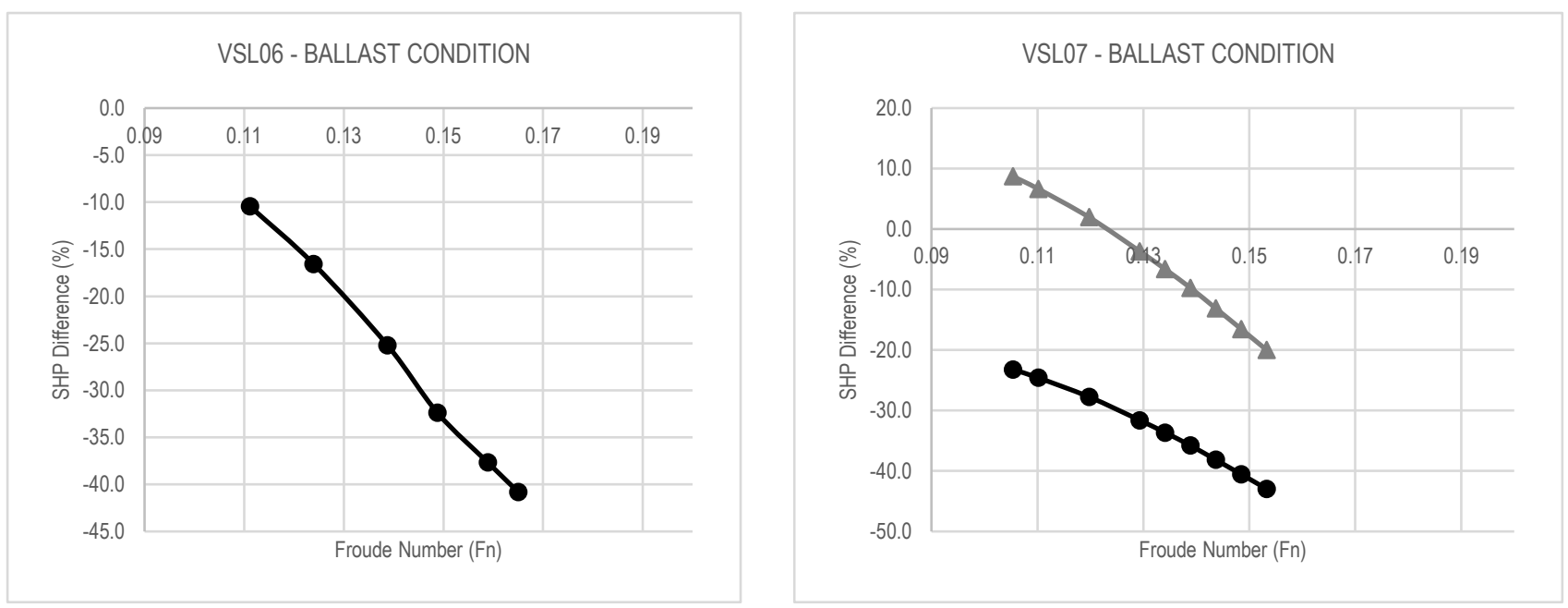

Figures [28] to [34]: Shaft Power prediction error (\%) distribution over different speeds - BALLAST Condition

The assumption for positive and negative difference is the same as in the case of EHP. From the above figures the following interesting result can be drawn.

Laden Condition

1. The strong correlation to the Froude number is still evident. However, for the case of SHP it is non-linear (in contrast to the case of EHP), illustrating a logarithmic trend for VSL03, 04, 05, 06 and 07 while for vessel VSL02 it is additionally fluctuating.

2. For 4 out of 10 total cases (40\%) there is an underestimation of the SHP for the entire Froude number range which increases with increasing Froude numbers. The underestimation ranges from $1 \%$ at lower speeds to maximum $10 \%$ at higher speeds ( 15 knots).

3. For 6 cases $(60 \%)$ there is an overestimation of maximum $17 \%$ at low Froude numbers which is decreasing by increasing Fn up to the range of 0.135 to $0.175 \mathrm{Fn}$ from which point there is an underestimation of the SHP increasing with the increase of the Froude number.

Ballast Condition

In ballast condition for all cases the same under prediction found in the EHP estimation was also evident here but on a smaller magnitude ranging from $10 \%$ at small Froude numbers up to $40 \%$ for high Froude numbers (close to 0.175 ). The trend of increasing under prediction by increasing speed is the same as in the case of the EHP.

From the above analysis, we can herein consider that the Holtrop and Mennen methodology is quite accurate for the EHP prediction but lacking accuracy in the SHP prediction as well as in the Ballast Conditions EHP and SHP prediction (off design condition). Furthermore, the evident correlation to Froude number (thus vessel's speed) and inaccuracy according to the Authors' perception can be attributed to the different flow development and phenomena which cannot be captured by an empirical method. The trends are very consistent thus underlining the result robustness.

\section{A Multi Staged Evolutionary Approach}

In order to be able to control the process of calibration as well as maintain the same ranking and composition of the total power requirements a multi-stage approach was adopted for the calibration, with each stage being treated as a typical optimization problem as depicted in Figure [35]. The constraints are part of the optimization, penalizing the non-feasible solutions and pushing for more feasible ones? 


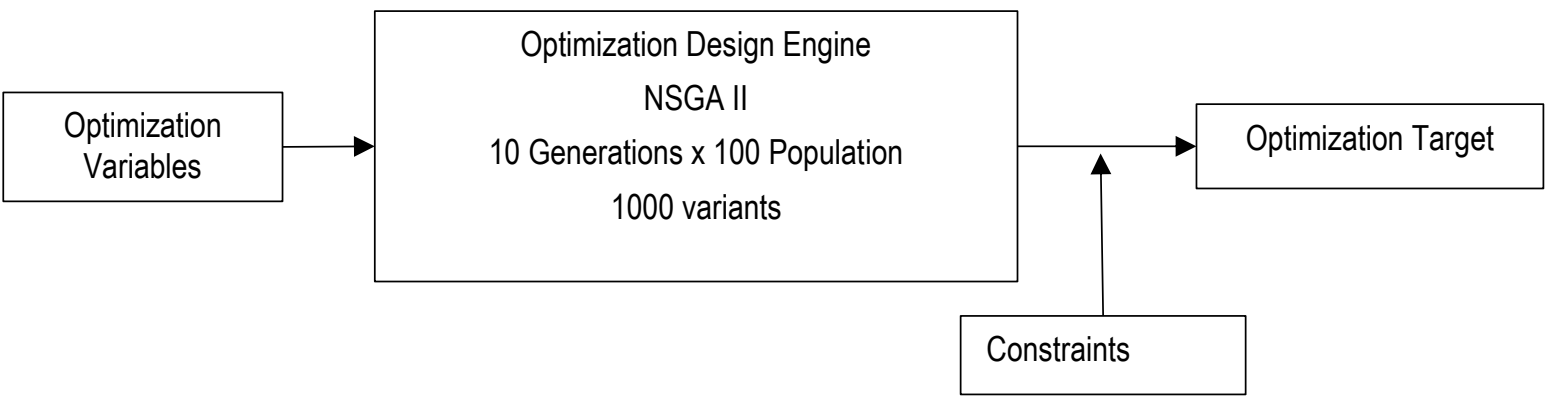

$\frac{1}{2}$

3

4

5

6

7

8

9

10

11

12

13

14

15

16

17

18

19

20

21

Figure [35]: Generalized optimization problem workflow

As in every optimization problem the following components were considered:

a. Optimization Variables

The variables of this problem were the selected constants and coefficients for each formula of each resistance component as formulated in the original Holtrop publication. The methodology was parametrically programmed and the constants became the variables of the new problem. The selected variables for the optimization can be summarized in the below table [2] for the laden condition and table [3] for the ballast condition respectively. 


\begin{tabular}{|c|c|c|c|c|}
\hline \multicolumn{5}{|c|}{ Calibration Variables - LADEN (Design and Scantling) Condition } \\
\hline No. & Constant in Holtrop Formula & $\begin{array}{r}\text { Value in Original } \\
\text { Holtrop } \\
\text { Publication } \\
\end{array}$ & $\begin{array}{r}\text { Value } \\
\begin{array}{r}\text { Calibration } \\
\text { Minimum }\end{array} \\
\end{array}$ & $\begin{array}{r}\text { Value } \\
\text { Calibration } \\
\text { Maximum } \\
\end{array}$ \\
\hline \multicolumn{5}{|c|}{ Wave Making Resistance } \\
\hline L-1 & $c_{1}=2223105 * c_{7}^{3.78613} *\left(\frac{T}{B}\right)^{1.07961} *\left(90-i_{E}\right)^{-1.37565}$ & 2223105 & 2200000 & 2300000 \\
\hline L-2 & $c_{1}=2223105 * c_{7}^{3.78613} *\left(\frac{T}{B}\right)^{1.07961} *\left(90-i_{E}\right)^{-1.37565}$ & 3.78613 & 2.0 & 4.70 \\
\hline L-3 & $c_{1}=2223105 * c_{7}^{3.78613} *\left(\frac{T}{B}\right)^{1.07961} *\left(90-i_{E}\right)^{-1.37565}$ & 1.07961 & 0.6 & 1.30 \\
\hline L-4 & $c_{1}=2223105 * c_{7}^{3.78613} *\left(\frac{T}{B}\right)^{1.07961} *\left(90-i_{E}\right)^{-1.37565}$ & -1.37565 & -2.0 & -0.80 \\
\hline L-5 & $c_{2}=\exp \left(-1.89 * \sqrt{c_{3}}\right)$ & -1.89 & -3.0 & -0.9 \\
\hline L-6 & $c_{3}=\frac{0.56 * A_{B T}^{1.5}}{B * T *\left(0.31 * \sqrt{A_{B T}}+T_{F}-h_{B}\right)}$ & 0.56 & 0.20 & 0.90 \\
\hline L-7 & $c_{3}=\frac{0.56 * A_{B T}}{B * T *\left(0.31 * \sqrt{A_{B T}}+T_{F}-h_{B}\right)}$ & 0.31 & 0.01 & 0.80 \\
\hline L-8 & $c_{3}=\frac{0.56 * A_{B T}}{B * T *\left(0.31 * \sqrt{A_{B T}}+T_{F}-h_{B}\right)}$ & 1.5 & 1.20 & 3.0 \\
\hline L-9 & $c_{5}=1-0.8 * \frac{A_{T}}{B * T * C_{M}}$ & 0.8 & 0.20 & 1.50 \\
\hline L-10 & $c_{15}=-1.69385+c_{15_{\text {new }}} * \frac{L^{3}}{\mathrm{~V}}$ & -1.69835 & -2.0 & -1.10 \\
\hline $\mathrm{L}-11$ & $c_{15}=-1.69385+c_{15_{\text {new }}} * \frac{L^{3}}{\mathrm{~V}}$ & $c_{15_{\text {new }}}=0$ & $\begin{array}{l}c_{15_{\text {new }}} \\
=-1\end{array}$ & $c_{15_{\text {new }}}=2$ \\
\hline L-12 & $m_{1}=0.0140407 * \frac{L}{T}-1.75254 * \frac{\nabla^{\frac{1}{3}}}{L}+4.79323 * \frac{B}{L}-c_{16}$ & 0.0140407 & 0.005 & 0.10 \\
\hline L-13 & $m_{1}=0.0140407 * \frac{L}{T}-1.75254 * \frac{\nabla^{\frac{1}{3}}}{L}+4.79323 * \frac{B}{L}-c_{16}$ & 1.75254 & 1.10 & 2.50 \\
\hline L-14 & $m_{1}=0.0140407 * \frac{L}{T}-1.75254 * \frac{\nabla^{\frac{1}{3}}}{L}+4.79323 * \frac{B}{L}-c_{16}$ & 4.79323 & 3.70 & 5.70 \\
\hline L-15 & $m_{2}=c_{15} * C_{p}^{2} * \exp \left(-0.1 * F n^{-2}\right)$ & 2 & 1.20 & 4.0 \\
\hline L-16 & $m_{2}=c_{15} * C_{p}{ }^{2} * \exp \left(-0.1 * F n^{-2}\right)$ & -0.1 & -0.50 & -0.04 \\
\hline L-17 & $m_{2}=c_{15} * C_{p}^{2} * \exp \left(-0.1 * F n^{-2}\right)$ & -2 & -4.0 & -1.20 \\
\hline L-18 & $c_{16}=1.73014-0.7067 * C_{P}+c_{16 C_{\text {new }}} *{C_{P}}^{2}+c_{16 D_{\text {new }}} * C_{P}{ }^{3}$ & 1.73014 & 1.20 & 2.50 \\
\hline L-19 & $c_{16}=1.73014-0.7067 * C_{P}+c_{16 C_{\text {new }}} *{C_{P}}^{2}+c_{16 D_{\text {new }}} * C_{P}{ }^{3}$ & 0.7067 & 0.20 & 1.20 \\
\hline L-20 & $c_{16}=1.73014-0.7067 * C_{P}+c_{16 C_{\text {new }}} *{C_{P}}^{2}+c_{16 D_{\text {new }}} *{C_{P}}^{3}$ & $c_{16 C_{\text {new }}}=0$ & $\begin{array}{l}c_{16 c_{\text {new }}} \\
=-2\end{array}$ & $c_{16 C_{\text {new }}}=2$ \\
\hline L-21 & $c_{16}=1.73014-0.7067 * C_{P}+c_{16 C_{\text {new }}} *{C_{P}}^{2}+c_{16 D_{\text {new }}} *{C_{P}}^{3}$ & $c_{16 D_{n e w}}=0$ & $\begin{array}{l}c_{16 C_{\text {new }}} \\
=-2\end{array}$ & $c_{16 C_{\text {new }}}=2$ \\
\hline L-22 & $\lambda=1.446 * C_{p}-0.03 * \frac{L}{B}+c_{n e w}$ & 1.446 & 0.60 & 2.0 \\
\hline L-23 & $\lambda=1.446 * C_{p}-0.03 * \frac{L}{B}+c_{\text {new }}$ & 0.03 & 0.01 & 0.10 \\
\hline L-24 & $\lambda=1.446 * C_{p}-0.03 * \frac{L}{B}+c_{n e w}$ & 0 & -2 & 2 \\
\hline L-25 & $d=-0.9$ & -0.9 & -1.50 & -0.30 \\
\hline L-26 & $R_{w}=c_{1} * c_{2} * c_{5} * \nabla * \rho * g * \exp \left\{m_{1} * F n^{d}+m_{2} * \cos \left(\lambda * F n^{-2}\right)\right\}$ & -2 & 0 & 1 \\
\hline \multicolumn{5}{|c|}{ Frictional Resistance - Form Factor } \\
\hline L-27 & $c_{12}=(T / L)^{0.2228446}$ & 0.2228446 & 0.05 & 0.80 \\
\hline
\end{tabular}




\begin{tabular}{|c|c|c|c|c|}
\hline L-28 & $\begin{array}{c}\left(1+k_{1}\right)=c_{13} *\left\{0.93+c_{12} *\left(\frac{B}{L_{R}}\right)^{0.92497} *\left(0.95-C_{P}\right)^{-0.521448}\right. \\
\left.*\left(1-C_{P}+0.0225 * L C B\right)^{0.6906}\right\}\end{array}$ & 0.92497 & 0.40 & 1.50 \\
\hline L-29 & $\begin{array}{c}\left(1+k_{1}\right)=c_{13} *\left\{0.93+c_{12} *\left(\frac{B}{L_{R}}\right)^{0.92497} *\left(0.95-C_{P}\right)^{-0.521448}\right. \\
\left.*\left(1-C_{P}+0.0225 * L C B\right)^{0.6906}\right\}\end{array}$ & -0.521448 & -0.90 & -0.10 \\
\hline L-30 & $\begin{array}{c}\left(1+k_{1}\right)=c_{13} *\left\{0.93+c_{12} *\left(\frac{B}{L_{R}}\right)^{0.92497} *\left(0.95-C_{P}\right)^{-0.521448}\right. \\
\left.*\left(1-C_{P}+0.0225 * L C B\right)^{0.6906}\right\}\end{array}$ & 0.6906 & 0.6906 & 1.50 \\
\hline L-31 & $c_{13}=1+0.003 * C_{\text {stern }}$ & 0 & -3 & 3 \\
\hline \multicolumn{5}{|c|}{ Resistance due to Bulbous Bow } \\
\hline L-32 & $P_{B}=\frac{0.56 * \sqrt{A_{B T}}}{T_{F}-1.5 * h_{B}}$ & 0.56 & 0.10 & 1.0 \\
\hline L-33 & $R_{B}=\frac{0.11 * \exp \left(-3 * P_{B}^{-2}\right) * F n i^{3} * A_{B T}{ }^{1.5} * \rho * g}{1+F n i^{2}}$ & 0.11 & 0.05 & 0.30 \\
\hline L-34 & $R_{B}=\frac{0.11 * \exp \left(-3 * P_{B}^{-2}\right) * F n i^{3} * A_{B T}^{1.5} * \rho * g}{1+F n i^{2}}$ & -3 & -5.0 & -1.50 \\
\hline L-35 & $R_{B}=\frac{0.11 * \exp \left(-3 * P_{B}^{-2}\right) * F n i^{3} * A_{B T}^{1.5} * \rho * g}{1+F n i^{2}}$ & 2 & 1.2 & 4 \\
\hline L-36 & $R_{B}=\frac{0.11 * \exp \left(-3 * P_{B}^{-2}\right) * F n i^{3} * A_{B T}^{1.5} * \rho * g}{1+F n i^{2}}$ & 3 & 1.5 & 4.0 \\
\hline L-37 & $R_{B}=\frac{0.11 * \exp \left(-3 * P_{B}{ }^{-2}\right) * F n i^{3} * A_{B T}^{1.5} * \rho * g}{1+F n i^{2}}$ & 1.5 & 1.20 & 3.0 \\
\hline L-38 & $R_{B}=\frac{0.11 * \exp \left(-3 * P_{B}^{-2}\right) * F n i^{3} * A_{B T}^{1.5} * \rho * g}{1+F n i^{2}}$ & 2 & 1.50 & 4.50 \\
\hline L-39 & $F n i=\frac{v}{\sqrt{g *\left(T_{F}-h_{B}-0.25 * \sqrt{A_{B T}}\right)+0.15 * v^{2}}}$ & 2 & 1.50 & 4.50 \\
\hline \multicolumn{5}{|c|}{ Resistance due to Transom Immersion } \\
\hline L-40 & $c_{6}=0.2 *\left(1-0.2 * F n_{T}\right)$ & 0.2 & 0.01 & 0.30 \\
\hline \multicolumn{5}{|c|}{ Model Ship Correlation } \\
\hline L-41 & $\begin{array}{c}C_{A}=0.006 *(L+100)^{-0.16}-0.00205+0.003 * \sqrt{\frac{L}{7.5}} * C_{B}^{4} * c_{2} *\left(0.04-c_{4}\right) \\
+c_{\text {Anew }} * F n^{c_{\text {Bnew }}}\end{array}$ & 0.006 & 0.001 & 0.10 \\
\hline L-42 & $\begin{array}{c}C_{A}=0.006 *(L+100)^{-0.16}-0.00205+0.003 * \sqrt{\frac{L}{7.5}} * C_{B}^{4} * C_{2} *\left(0.04-c_{4}\right) \\
+c_{\text {Anew }} * F n^{c_{\text {Bnew }}}\end{array}$ & -0.16 & -0.50 & -0.01 \\
\hline L-43 & $\begin{array}{c}C_{A}=0.006 *(L+100)^{-0.16}-0.00205+0.003 * \sqrt{\frac{L}{7.5}} * C_{B}{ }^{4} * c_{2} *\left(0.04-c_{4}\right) \\
+c_{\text {Anew }} * F n^{c_{\text {Bnew }}}\end{array}$ & -0.00205 & -0.10 & -0.0001 \\
\hline L-44 & $\begin{array}{c}C_{A}=0.006 *(L+100)^{-0.16}-0.00205+0.003 * \sqrt{\frac{L}{7.5}} * C_{B}{ }^{4} * c_{2} *\left(0.04-c_{4}\right) \\
+c_{\text {Anew }} * F n^{c_{\text {Bnew }}}\end{array}$ & 0.003 & 0.001 & 0.10 \\
\hline
\end{tabular}




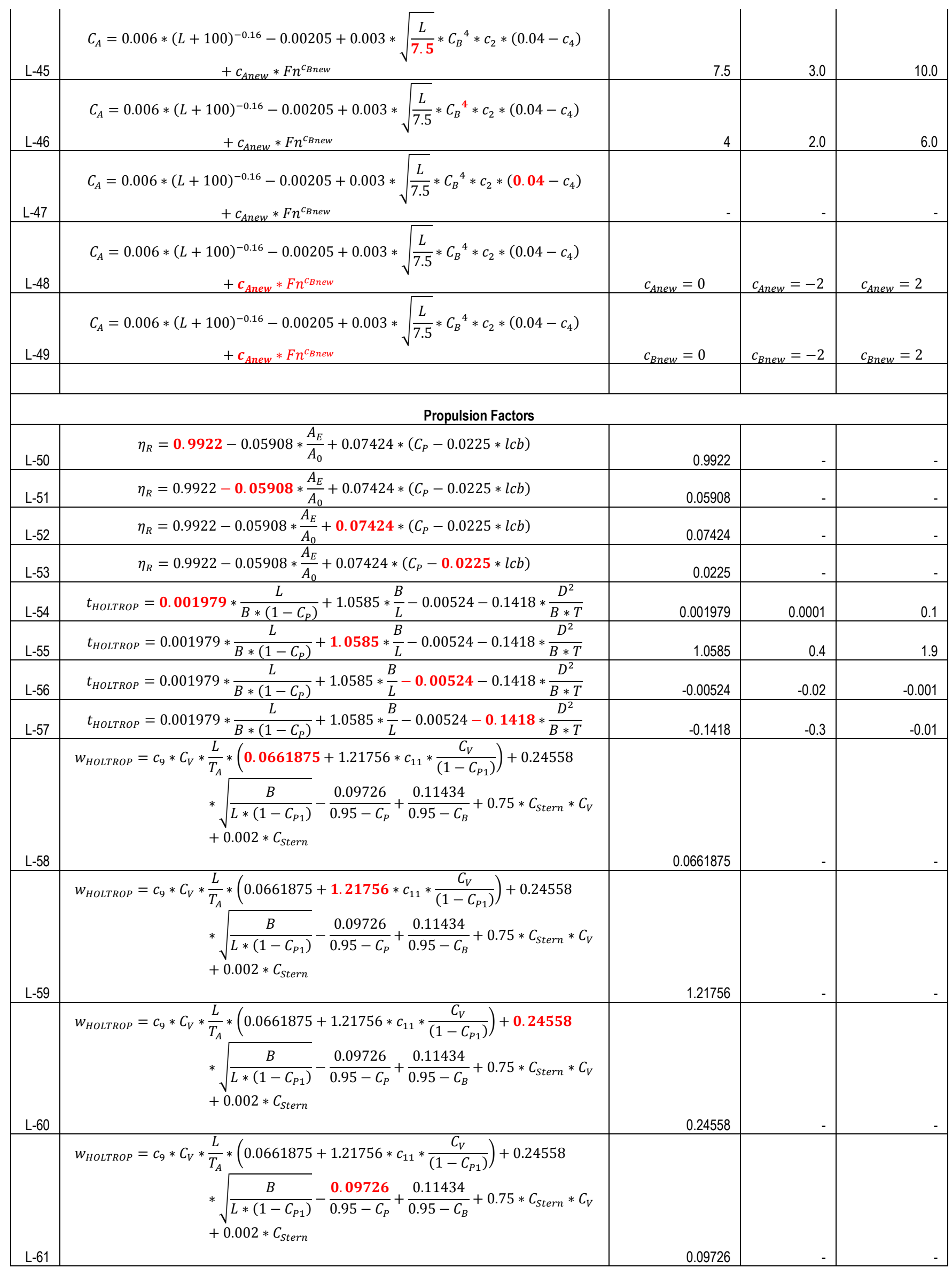




\begin{tabular}{|c|c|c|c|c|}
\hline L-62 & $\begin{aligned} w_{\text {HOLTROP }}=c_{9} * C_{V} * & \frac{L}{T_{A}} *\left(0.0661875+1.21756 * c_{11} * \frac{C_{V}}{\left(1-C_{P 1}\right)}\right)+0.24558 \\
& * \sqrt{\frac{B}{L *\left(1-C_{P 1}\right)}}-\frac{0.09726}{0.95-C_{P}}+\frac{\mathbf{0 . 1 1 4 3 4}}{0.95-C_{B}}+0.75 * C_{\text {Stern }} * C_{V} \\
& +0.002 * C_{\text {Stern }}\end{aligned}$ & 0.11434 & - & - \\
\hline L-63 & $c_{11}=0.0833333 *\left(\frac{T_{A}}{D}\right)^{3}+1.33333$ & 0.08333333 & - & - \\
\hline L-64 & $c_{11}=0.0833333 *\left(\frac{T_{A}}{D}\right)^{3}+1.33333$ & 1.3333 & - & - \\
\hline L-65 & $w_{S C H N E E K L U T H}=0.5 * C_{P} * \frac{1.6}{1+\frac{D}{T}} * \frac{16}{10+\frac{L}{B}}$ & 0.5 & 0.1 & 0.9 \\
\hline L-66 & $w_{\text {SCHNEEKLUTH }}=0.5 * C_{P} * \frac{1.6}{1+\frac{D}{T}} * \frac{16}{10+\frac{L}{B}}$ & 1.6 & 0.1 & 3 \\
\hline L-67 & $w_{\text {SCHNEEKLUTH }}=0.5 * C_{P} * \frac{1.6}{1+\frac{D}{T}} * \frac{16}{10+\frac{L}{B}}$ & 16 & 8 & 20 \\
\hline L-68 & $w_{\text {KRUGER }}=0.75 * C_{B}-0.24$ & 0.75 & 0.5 & 1.5 \\
\hline L-69 & $w_{\text {KRUGER }}=0.75 * C_{B}-\mathbf{0 . 2 4}$ & 0.24 & 0.1 & 0.5 \\
\hline L-70 & $w_{H E C K S C H E R}=0.7 * C_{P}-0.18$ & 0.7 & 0.25 & 1.5 \\
\hline L-71 & $w_{H E C K S C H E R}=0.7 * C_{P}-\mathbf{0 . 1 8}$ & -0.18 & -0.3 & 0.3 \\
\hline L-72 & $w_{T R O O S T}=0.25+2.5 *\left(C_{B}-0.6\right)^{2}$ & 0.25 & 0.1 & 0.4 \\
\hline L-73 & $w_{T R O O S T}=0.25+2.5 *\left(C_{B}-0.6\right)^{2}$ & 2.5 & 0.1 & 4 \\
\hline
\end{tabular}

1 Table [2]: Holtrop Constants used as variables in optimization problem and their respective value range - LADEN

2

CONDITION.

3

4

\begin{tabular}{|c|c|c|c|c|}
\hline \multicolumn{5}{|c|}{ Calibration Variables - BALLAST Condition } \\
\hline No. & Constant in Holtrop Formula & $\begin{array}{r}\text { Value in Original } \\
\text { Holtrop } \\
\text { Publication }\end{array}$ & $\begin{array}{r}\text { Value } \\
\text { Calibration } \\
\text { Minimum }\end{array}$ & $\begin{array}{r}\text { Value Calibration } \\
\text { Maximum }\end{array}$ \\
\hline \multicolumn{5}{|c|}{ Wave Making Resistance } \\
\hline B-1 & $c_{1}=2223105 * c_{7}^{3.78613} *\left(\frac{T}{B}\right)^{1.07961} *\left(90-i_{E}\right)^{-1.37565}$ & 2223105 & 2100000 & 2250000 \\
\hline B-2 & $c_{1}=2223105 * c_{7}^{3.78613} *\left(\frac{T}{B}\right)^{1.07961} *\left(90-i_{E}\right)^{-1.37565}$ & 3.78613 & 3.6 & 3.8 \\
\hline B-3 & $c_{1}=2223105 * c_{7}^{3.78613} *\left(\frac{T}{B}\right)^{1.07961} *\left(90-i_{E}\right)^{-1.37565}$ & 1.07961 & 1.0 & 1.2 \\
\hline B-4 & $c_{1}=2223105 * c_{7}^{3.78613} *\left(\frac{T}{B}\right)^{1.07961} *\left(90-i_{E}\right)^{-1.37565}$ & -1.37565 & -1.5 & -1.0 \\
\hline B-5 & $c_{2}=\exp \left(-1.89 * \sqrt{c_{3}}\right)$ & -1.89 & -2.5 & -1.5 \\
\hline B-6 & $c_{3}=\frac{0.56 * A_{B T}^{1.5}}{B * T *\left(0.31 * \sqrt{A_{B T}}+T_{F}-h_{B}\right)}$ & 0.56 & 0.40 & 0.60 \\
\hline B-7 & $c_{3}=\frac{0.56 * A_{B T}}{B * T *\left(0.31 * \sqrt{A_{B T}}+T_{F}-h_{B}\right)}$ & 0.31 & 0.20 & 0.40 \\
\hline B-8 & $c_{3}=\frac{0.56 * A_{B T}}{B * T *\left(0.31 * \sqrt{A_{B T}}+T_{F}-h_{B}\right)}$ & 1.5 & 1.2 & 3 \\
\hline B-9 & $c_{5}=1-0.8 * \frac{A_{T}}{B * T * C_{M}}$ & 0.8 & 0.7 & 0.9 \\
\hline B-10 & $c_{15}=-1.69385+c_{15_{\text {new }}} * \frac{L^{3}}{\mathrm{~V}}$ & -1.69835 & -1.80 & -1.50 \\
\hline B-11 & $c_{15}=-1.69385+c_{15_{\text {new }}} * \frac{L^{3}}{\mathrm{~V}}$ & $c_{15_{\text {new }}}=0$ & $c_{15_{\text {new }}}=-1$ & $c_{15_{\text {new }}}=3$ \\
\hline B-12 & $m_{1}=0.0140407 * \frac{L}{T}-1.75254 * \frac{\nabla^{\frac{1}{3}}}{L}+4.79323 * \frac{B}{L}-c_{16}$ & 0.0140407 & 0.01 & 0.02 \\
\hline
\end{tabular}




\begin{tabular}{|c|c|c|c|c|}
\hline B-13 & $m_{1}=0.0140407 * \frac{L}{T}-1.75254 * \frac{\nabla^{\frac{1}{3}}}{L}+4.79323 * \frac{B}{L}-c_{16}$ & 1.75254 & 1.60 & 1.90 \\
\hline B-14 & $m_{1}=0.0140407 * \frac{L}{T}-1.75254 * \frac{\nabla^{\frac{1}{3}}}{L}+4.79323 * \frac{B}{L}-c_{16}$ & 4.79323 & 4.60 & 4.90 \\
\hline B-15 & $m_{2}=c_{15} * C_{p}^{2} * \exp \left(-0.1 * F n^{-2}\right)$ & 2 & 1.2 & 3 \\
\hline B-16 & $m_{2}=c_{15} * C_{p}^{2} * \exp \left(-0.1 * F n^{-2}\right)$ & -0.1 & -0.8 & -0.01 \\
\hline B-17 & $m_{2}=c_{15} * C_{p}^{2} * \exp \left(-0.1 * F n^{-2}\right)$ & -2 & -3.5 & -1.5 \\
\hline B-18 & $c_{16}=8.07981 * C_{P}-13.8673 * C_{P}^{2}+6.984388 * C_{P}{ }^{3}$ & 8.07981 & 4.0 & 12.0 \\
\hline B-19 & $c_{16}=8.07981 * C_{P}-13.8673 * C_{P}{ }^{2}+6.984388 * C_{P}{ }^{3}$ & -13.8673 & -25.0 & -10.0 \\
\hline B-20 & $c_{16}=8.07981 * C_{P}-13.8673 * C_{P}{ }^{2}+6.984388 * C_{P}{ }^{3}$ & 6.984388 & 3.0 & 11.0 \\
\hline B-21 & $c_{16}=8.07981 * C_{P}-13.8673 * C_{P}{ }^{2}+6.984388 * C_{P}{ }^{3}$ & 1 & 0.9 & 1.6 \\
\hline B-22 & $c_{16}=8.07981 * C_{P}-13.8673 * C_{P}{ }^{2}+6.984388 * C_{P}{ }^{3}$ & 2 & 1.8 & 2.9 \\
\hline B-23 & $c_{16}=8.07981 * C_{P}-13.8673 * C_{P}{ }^{2}+6.984388 * C_{P}{ }^{3}$ & 3 & 2.6 & 3.5 \\
\hline B-24 & $\lambda=1.446 * C_{p}-0.03 * \frac{L}{B}+c_{\text {new }}$ & 1.446 & 1.40 & 1.50 \\
\hline B-25 & $\lambda=1.446 * C_{p}-0.03 * \frac{L}{B}+c_{n e w}$ & 0.03 & 0.001 & 1.0 \\
\hline B-26 & $\lambda=1.446 * C_{p}-0.03 * \frac{L}{B}+c_{\text {new }}$ & 0 & -2.0 & 2.0 \\
\hline B-27 & $d=-0.9$ & -0.9 & -2.0 & -0.1 \\
\hline B-28 & $R_{w}=c_{1} * c_{2} * c_{5} * \nabla * \rho * g * \exp \left\{m_{1} * F n^{d}+m_{2} * \cos \left(\lambda * F n^{-2}\right)\right\}$ & -2 & -3.0 & 1.50 \\
\hline \multicolumn{5}{|c|}{ Frictional Resistance - Form Factor } \\
\hline B-29 & $c_{12}=48.20 *\left(\frac{T}{L}-0.02\right)^{2.078}+0.479948$ & 48.20 & 25.0 & 60.0 \\
\hline B-30 & $c_{12}=48.20 *\left(\frac{T}{L}-0.02\right)^{2.078}+0.479948$ & 2.078 & 0.50 & 4.0 \\
\hline B-31 & $c_{12}=48.20 *\left(\frac{T}{L}-0.02\right)^{2.078}+0.479948$ & 0.479948 & 0.35 & 0.60 \\
\hline B-32 & $\begin{array}{c}\left(1+k_{1}\right)=c_{13} *\left\{0.93+c_{12} *\left(\frac{B}{L_{R}}\right)^{0.92497} *\left(0.95-C_{P}\right)^{-0.521448}\right. \\
\left.*\left(1-C_{P}+0.0225 * L C B\right)^{0.6906}\right\}\end{array}$ & 0.92497 & 0.30 & 1.50 \\
\hline B-33 & $\begin{array}{c}\left(1+k_{1}\right)=c_{13} *\left\{0.93+c_{12} *\left(\frac{B}{L_{R}}\right)^{0.92497} *\left(0.95-C_{P}\right)^{-0.521448}\right. \\
\left.*\left(1-C_{P}+0.0225 * L C B\right)^{0.6906}\right\}\end{array}$ & -0.521448 & -3.0 & -1.50 \\
\hline B-34 & $\begin{array}{c}\left(1+k_{1}\right)=c_{13} *\left\{0.93+c_{12} *\left(\frac{B}{L_{R}}\right)^{0.92497} *\left(0.95-C_{P}\right)^{-0.521448}\right. \\
\left.*\left(1-C_{P}+0.0225 * L C B\right)^{0.6906}\right\}\end{array}$ & 0.6906 & 0.20 & 0.90 \\
\hline B-35 & $c_{13}=1+0.003 * C_{\text {stern }}$ & 0.003 & 0.001 & 0.70 \\
\hline B-36 & $c_{13}=1+0.003 * C_{\text {stern }}$ & 0 & 0.10 & 2.0 \\
\hline \multicolumn{5}{|c|}{ Resistance due to Bulbous Bow } \\
\hline B-37 & $P_{B}=\frac{0.56 * \sqrt{A_{B T}}}{T_{F}-1.5 * h_{B}}$ & 0.56 & 0.10 & 0.90 \\
\hline B-38 & $R_{B}=\frac{0.11 * \exp \left(-3 * P_{B}{ }^{-2}\right) * F n i^{3} * A_{B T}^{1.5} * \rho * g}{1+F n i^{2}}$ & 0.11 & 0.01 & 0.30 \\
\hline & $R_{B}=\frac{0.11 * \exp \left(-3 * P_{B}^{-2}\right) * F n i^{3} * A_{B T}^{1.5} * \rho * g}{1+F n i^{2}}$ & & & \\
\hline B-39 & & -3 & -5.0 & -1.0 \\
\hline
\end{tabular}




\begin{tabular}{|c|c|c|c|c|}
\hline B-40 & $R_{B}=\frac{0.11 * \exp \left(-3 * P_{B}{ }^{-2}\right) * F n i^{3} * A_{B T}^{1.5} * \rho * g}{1+F n i^{2}}$ & 2 & -3.0 & -1.50 \\
\hline & $R_{B}=\frac{0.11 * \exp \left(-3 * P_{B}^{-2}\right) * F n i^{3} * A_{B T}{ }^{1.5} * \rho * g}{1+F n i^{2}}$ & \multirow[b]{2}{*}{3} & \multirow[b]{2}{*}{1.80} & \multirow[b]{2}{*}{4.0} \\
\hline B-41 & & & & \\
\hline & $R_{B}=\frac{0.11 * \exp \left(-3 * P_{B}^{-2}\right) * F n i^{3} * A_{B T}{ }^{1.5} * \rho * g}{1+F n i^{2}}$ & & & \\
\hline B-42 & & 1.5 & 1.2 & 3.0 \\
\hline B-43 & $R_{B}=\frac{0.11 * \exp \left(-3 * P_{B}{ }^{-2}\right) * F n i^{3} * A_{B T}^{1.5} * \rho * g}{1+F n i^{2}}$ & 2 & 1.60 & 2.60 \\
\hline B-44 & $F n i=\frac{v}{\sqrt{g *\left(T_{F}-h_{B}-0.25 * \sqrt{A_{B T}}\right)+0.15 * v^{2}}}$ & 2 & 1.40 & 2.60 \\
\hline \multicolumn{5}{|c|}{ Resistance due to Transom Immersion } \\
\hline B-45 & $c_{6}=0.2 *\left(1-0.2 * F n_{T}\right)$ & 0.2 & 0.01 & 2.0 \\
\hline \multicolumn{5}{|c|}{ Model Ship Correlation } \\
\hline & $\begin{array}{c}C_{A}=0.006 *(L+100)^{-0.16}-0.00205+0.003 * \sqrt{\frac{L}{7.5} * C_{B}{ }^{4} * c_{2} *\left(0.04-c_{4}\right)} \\
+c_{\text {Anew }} * F n^{c_{B n e w}}\end{array}$ & & & \\
\hline B-46 & & 0.006 & 0.001 & 0.50 \\
\hline B-47 & $\begin{array}{c}C_{A}=0.006 *(L+100)^{-0.16}-0.00205+0.003 * \sqrt{\frac{L}{7.5} * C_{B}{ }^{4} * C_{2} *\left(0.04-c_{4}\right)} \\
+c_{\text {Anew }} * F n^{c_{\text {Bnew }}}\end{array}$ & -0.16 & -0.50 & -0.01 \\
\hline B-48 & $\begin{array}{c}C_{A}=0.006 *(L+100)^{-0.16}-0.00205+0.003 * \sqrt{\frac{L}{7.5} * C_{B}{ }^{4} * c_{2} *\left(0.04-c_{4}\right)} \\
+c_{\text {Anew }} * F n^{c_{\text {Bnew }}}\end{array}$ & -0.00205 & -0.06 & -0.10 \\
\hline B-49 & $\begin{array}{c}C_{A}=0.006 *(L+100)^{-0.16}-0.00205+0.003 * \sqrt{\frac{L}{7.5} * C_{B}{ }^{4} * C_{2} *\left(0.04-c_{4}\right)} \\
+c_{\text {Anew }} * F n^{c_{\text {Bnew }}}\end{array}$ & 0.003 & 0.001 & 0.10 \\
\hline B-50 & $\begin{array}{c}C_{A}=0.006 *(L+100)^{-0.16}-0.00205+0.003 * \sqrt{\frac{L}{7.5} * C_{B}{ }^{4} * C_{2} *\left(0.04-c_{4}\right)} \\
+c_{\text {Anew }} * F n^{c_{\text {Bnew }}}\end{array}$ & 7.5 & 6.0 & 11.0 \\
\hline B-51 & $\begin{array}{c}C_{A}=0.006 *(L+100)^{-0.16}-0.00205+0.003 * \sqrt{\frac{L}{7.5}} * C_{B}{ }^{4} * c_{2} *\left(0.04-c_{4}\right) \\
+c_{\text {Anew }} * F n^{c_{\text {Bnew }}}\end{array}$ & 0.04 & - & - \\
\hline B-52 & $\begin{array}{c}C_{A}=0.006 *(L+100)^{-0.16}-0.00205+0.003 * \sqrt{\frac{L}{7.5} * C_{B}^{4} * C_{2} *\left(0.04-c_{4}\right)} \\
+c_{\text {Anew }} * F n^{c_{\text {Bnew }}}\end{array}$ & $c_{\text {Anew }}=0$ & $c_{\text {Anew }}=0.20$ & $c_{\text {Anew }}=1.20$ \\
\hline B-53 & $\begin{array}{c}C_{A}=0.006 *(L+100)^{-0.16}-0.00205+0.003 * \sqrt{\frac{L}{7.5}} * C_{B}^{4} * C_{2} *\left(0.04-c_{4}\right) \\
+c_{\text {Anew }} * F n^{c_{\text {Bnew }}}\end{array}$ & $c_{\text {Bnew }}=0$ & $c_{\text {Bnew }}=-1.0$ & $c_{\text {Bnew }}=1.0$ \\
\hline B-54 & $c_{4}=c_{4 n e w} * \frac{T_{F}}{L}$ & $c_{4 \text { new }}=1$ & $c_{4 \text { new }}=0.01$ & $c_{4 \text { new }}=5$ \\
\hline \multicolumn{5}{|c|}{$\begin{array}{ll}\text { Propulsion Factors } \\
\end{array}$} \\
\hline B-53 & $\eta_{R}=0.9922-0.05908 * \frac{A_{E}}{A_{0}}+0.07424 *\left(C_{P}-0.0225 * l c b\right)$ & 0.9922 & - & - \\
\hline B-54 & $\eta_{R}=0.9922-0.05908 * \frac{A_{E}}{A_{0}}+0.07424 *\left(C_{P}-0.0225 * l c b\right)$ & 0.05908 & - & - \\
\hline B-55 & $\eta_{R}=0.9922-0.05908 * \frac{A_{E}}{A_{0}}+0.07424 *\left(C_{P}-0.0225 * l c b\right)$ & 0.07424 & - & - \\
\hline B-56 & $\eta_{R}=0.9922-0.05908 * \frac{A_{E}}{A_{0}}+0.07424 *\left(C_{P}-0.0225 * l c b\right)$ & 0.0225 & - & - \\
\hline
\end{tabular}




\begin{tabular}{|c|c|c|c|c|}
\hline B-57 & $t_{\text {HOLTROP }}=0.001979 * \frac{L}{B *\left(1-C_{P}\right)}+1.0585 * \frac{B}{L}-0.00524-0.1418 * \frac{D^{2}}{B * T}$ & 0.001979 & 0.0001 & 0.9 \\
\hline B-58 & $t_{\text {HOLTROP }}=0.001979 * \frac{L}{B *\left(1-C_{P}\right)}+1.0585 * \frac{B}{L}-0.00524-0.1418 * \frac{D^{2}}{B * T}$ & 1.0585 & 0.5 & 2.5 \\
\hline B-59 & $t_{\text {HOLTROP }}=0.001979 * \frac{L}{B *\left(1-C_{P}\right)}+1.0585 * \frac{B}{L}-0.00524-0.1418 * \frac{D^{2}}{B * T}$ & -0.00524 & -0.1 & $-10^{-5}$ \\
\hline B-60 & $t_{\text {HOLTROP }}=0.001979 * \frac{L}{B *\left(1-C_{P}\right)}+1.0585 * \frac{B}{L}-0.00524-0.1418 * \frac{D^{2}}{B * T}$ & -0.1418 & 0.0001 & 0.9 \\
\hline B-61 & $\begin{aligned} w_{\text {HOLTROP }}=c_{9} * C_{V} * & \frac{L}{T_{A}} *\left(0.0661875+1.21756 * c_{11} * \frac{C_{V}}{\left(1-C_{P 1}\right)}\right)+0.24558 \\
& * \sqrt{\frac{B}{L *\left(1-C_{P 1}\right)}-\frac{0.09726}{0.95-C_{P}}+\frac{0.11434}{0.95-C_{B}}+0.75 * C_{\text {Stern }}} \\
& * C_{V}+0.002 * C_{\text {Stern }}\end{aligned}$ & 0.0661875 & 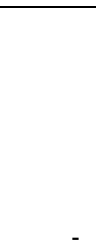 & - \\
\hline B-62 & $\begin{aligned} w_{\text {HOLTROP }}=c_{9} * C_{V} * & \frac{L}{T_{A}} *\left(0.0661875+1.21756 * c_{11} * \frac{C_{V}}{\left(1-C_{P 1}\right)}\right)+0.24558 \\
& * \sqrt{\frac{B}{L *\left(1-C_{P 1}\right)}}-\frac{0.09726}{0.95-C_{P}}+\frac{0.11434}{0.95-C_{B}}+0.75 * C_{\text {Stern }} \\
& * C_{V}+0.002 * C_{\text {Stern }}\end{aligned}$ & 1.21756 & - & - \\
\hline B-63 & $\begin{aligned} w_{\text {HOLTROP }}=c_{9} * C_{V} * & \frac{L}{T_{A}} *\left(0.0661875+1.21756 * c_{11} * \frac{C_{V}}{\left(1-C_{P 1}\right)}\right)+0.24558 \\
& * \sqrt{\frac{B}{L *\left(1-C_{P 1}\right)}}-\frac{0.09726}{0.95-C_{P}}+\frac{0.11434}{0.95-C_{B}}+0.75 * C_{\text {Stern }} \\
& * C_{V}+0.002 * C_{\text {Stern }}\end{aligned}$ & 0.24558 & - & - \\
\hline B-64 & $\begin{aligned} w_{\text {HOLTROP }}=c_{9} * C_{V} * & \frac{L}{T_{A}} *\left(0.0661875+1.21756 * c_{11} * \frac{C_{V}}{\left(1-C_{P 1}\right)}\right)+0.24558 \\
& * \sqrt{\frac{B}{L *\left(1-C_{P 1}\right)}}-\frac{\mathbf{0 . 0 9 7 2 6}}{0.95-C_{P}}+\frac{0.11434}{0.95-C_{B}}+0.75 * C_{\text {Stern }} \\
& * C_{V}+0.002 * C_{\text {Stern }}\end{aligned}$ & 0.09726 & - & - \\
\hline B-65 & $\begin{aligned} w_{\text {HOLTROP }}=c_{9} * C_{V} * & \frac{L}{T_{A}} *\left(0.0661875+1.21756 * c_{11} * \frac{C_{V}}{\left(1-C_{P 1}\right)}\right)+0.24558 \\
& * \sqrt{\frac{B}{L *\left(1-C_{P 1}\right)}}-\frac{0.09726}{0.95-C_{P}}+\frac{\mathbf{0 . 1 1 4 3 4}}{0.95-C_{B}}+0.75 * C_{\text {Stern }} \\
& * C_{V}+0.002 * C_{\text {Stern }}\end{aligned}$ & 011434 & - & - \\
\hline B-66 & $c_{11}=0.0833333 *\left(\frac{T_{A}}{D}\right)^{3}+1.33333$ & 0.08333333 & - & - \\
\hline B-67 & $c_{11}=0.0833333 *\left(\frac{T_{A}}{D}\right)^{3}+1.33333$ & 1.3333 & - & - \\
\hline B-68 & $w_{\text {SCHNEEKLUTH }}=0.5 * C_{P} * \frac{1.6}{1+\frac{D}{T}} * \frac{16}{10+\frac{L}{B}}$ & 0.5 & 0.1 & 0.9 \\
\hline B-69 & $w_{\text {SCHNEEKLUTH }}=0.5 * C_{P} * \frac{1.6}{1+\frac{D}{T}} * \frac{16}{10+\frac{L}{B}}$ & 1.6 & 0.5 & 2.5 \\
\hline B-70 & $w_{\text {SCHNEEKLUTH }}=0.5 * C_{P} * \frac{1.6}{1+\frac{D}{T}} * \frac{16}{10+\frac{L}{B}}$ & 16 & 6 & 25 \\
\hline B-71 & $w_{\text {KRUGER }}=0.75 * C_{B}-0.24$ & 0.75 & 0.4 & 0.9 \\
\hline B-72 & $w_{K R U G E R}=0.75 * C_{B}-\mathbf{0 . 2 4}$ & 0.24 & 0.05 & 0.5 \\
\hline B-73 & $w_{H E C K S C H E R}=0.7 * C_{P}-0.18$ & 0.7 & 0.2 & 0.9 \\
\hline B-74 & $w_{H E C K S C H E R}=0.7 * C_{P}-\mathbf{0 . 1 8}$ & -0.18 & -0.40 & -0.01 \\
\hline B-75 & $w_{\text {TROOST }}=0.25+2.5 *\left(C_{B}-0.6\right)^{2}$ & 0.25 & 0.1 & 0.4 \\
\hline B-76 & $w_{\text {TROOST }}=0.25+2.5 *\left(C_{B}-0.6\right)^{2}$ & 2.5 & 0.1 & 4 \\
\hline
\end{tabular}

1 Table [3]: Holtrop Constants used as variables in optimization problem and their respective value range - BALLAST

2 CONDITION. 


\section{b. Optimization Target}

For each vessel case the difference between the Holtrop prediction and the respective model test result for each speed run (basis on the model tests) is calculated and its minimization is set as the target of the optimization run. The difference is dependent on the calibration stage and can either be the difference in the Effective Power or the difference in the propulsion factors, namely the thrust deduction, relative rotative efficiency and wake field fraction respectively. It should be pointed out that the absolute value (i.e, unsigned magnitude) of the differences was used instead of the signed difference since using the latter might lead to results with larger overall errors if the positive and negative differences cancel each other.

\section{c. Design Engine}

The design engine applied can be either the NSGA II (Non-dominating Sorting Genetic Algorithm) [10] or the MOSA (Multi Objective Simulation Annealing) [11] algorithms. These are employed in the CAE software CAESES (ex-Friendship Framework) where the simulation and variation is programmed.

\section{d. Constraints}

The only constraint set was an upper limit on the optimization target, which is rejecting effectively combinations of the coefficients resulting into an average EHP and average SHP difference (depending on the calibration stage) greater than 15\%. This was done in order to put a restriction and since this is an evolutionary algorithm push the latter to converge to the target.

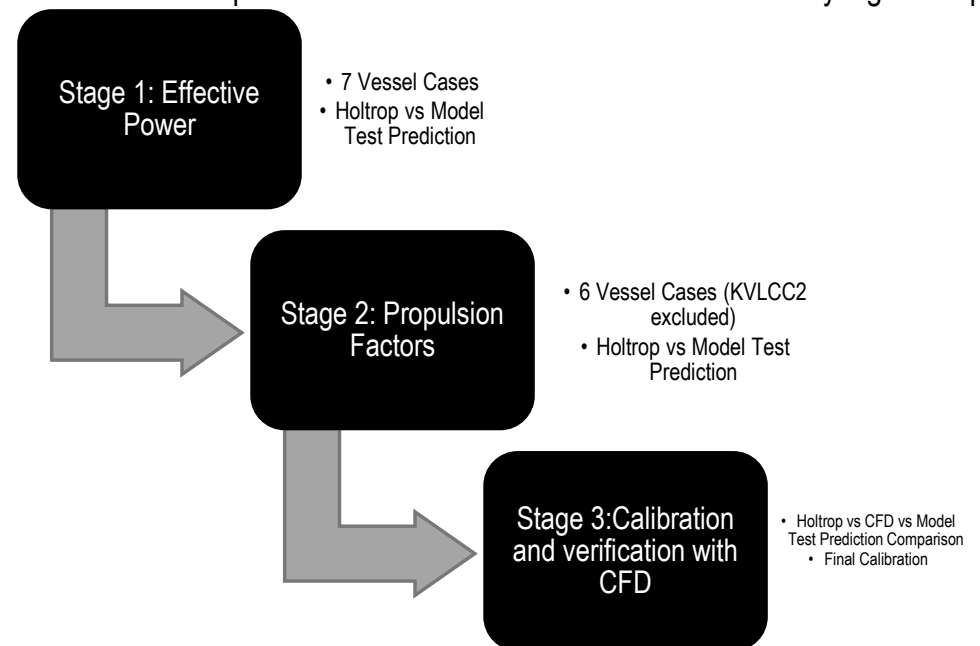

Figure [36]: Stages and tools employed for each one

\subsection{Stage 1 : Bare Hull Resistance Calibration}

The first calibration stage is for the bare hull resistance and power requirement. The parameters that underwent systematic variation were from 1 to 49 for Laden Conditions and 1 to 54 for Ballast Conditions are depicted in table [2] and table [3] with the same range of variance. The variation engine employed was the NSGA II algorithm in the CAESES platform with a chosen set of 10 generations of variants with each generation having 100 variants population thus resulting into 1000 variants, in other words 1000 combinations of the 49 parameters.

For this stage, the optimization target was set to be the absolute value of the percentage of difference between the Effective Horsepower (EHP-kW) between the model test estimation and each "Holtrop variant" prediction, with minimum being the desired merit. This was done for all 7 vessel cases in all speed-power points of each model test, resulting into 111 differences calculated at the points referred to as "calibration points". Furthermore, for the ease of selection process the average value of the differences of the calibration points per vessel was calculated as it can assist in the sorting of the best variants during post-processing. Within this spirit, the average value for the methodology in total (as average of the averages of absolute differences) was calculated in order to serve also as a constraint. This constraint imposed was set as having not more than $15 \%$ of average deviations.

The optimization was on a chain basis for 3 optimization loops, meaning that the best variant from the first run was set as the initial solution for the $2^{\text {nd }}$ and the best variant from the $2^{\text {nd }}$ run was set as the initial solution for the $3^{\text {rd }}$ run. This was done as the initial solution/state is critical for evolutionary algorithms such as the NSGAll contributing to more efficient convergence as well as a better final solution.

The results were sorted and a combination was selected from the lowest average absolute difference basis. The resulting values improved the Effective Power curves prediction error as it can be seen from the below Figures [34] to [47] and [48] to [54]. In all below graphs the grey, triangle marker curve represents the error distribution after calibration while the black, circle marker curve the error distribution corresponding to the original coefficients of the method. 
1

2
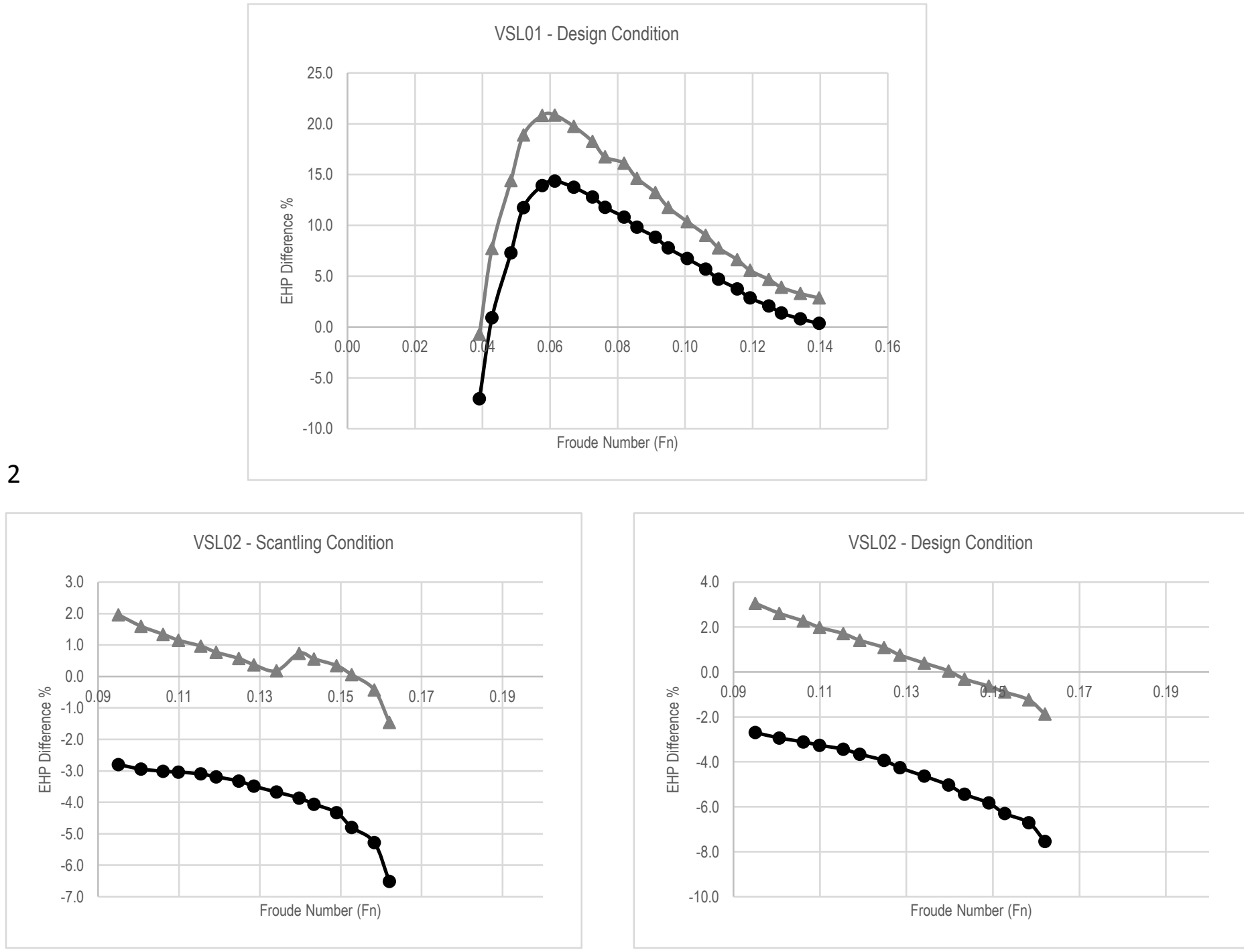

3
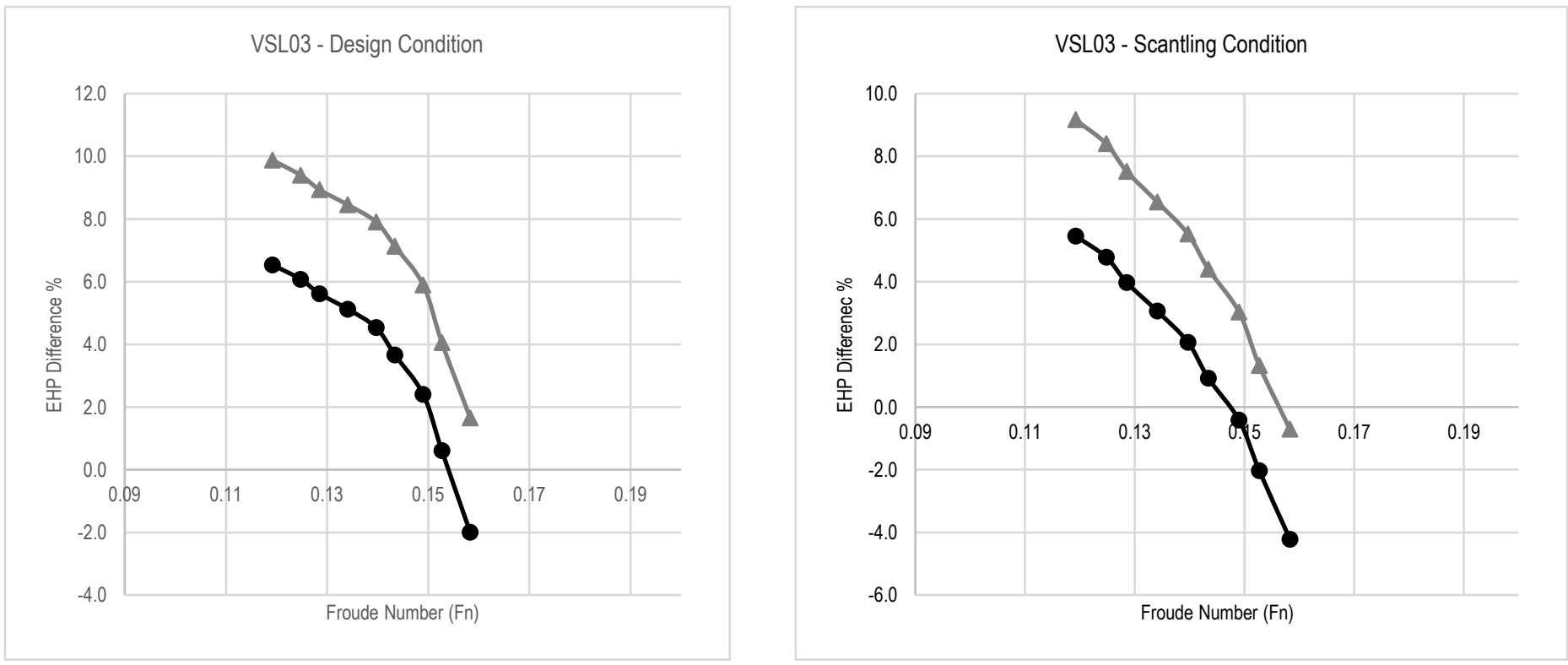

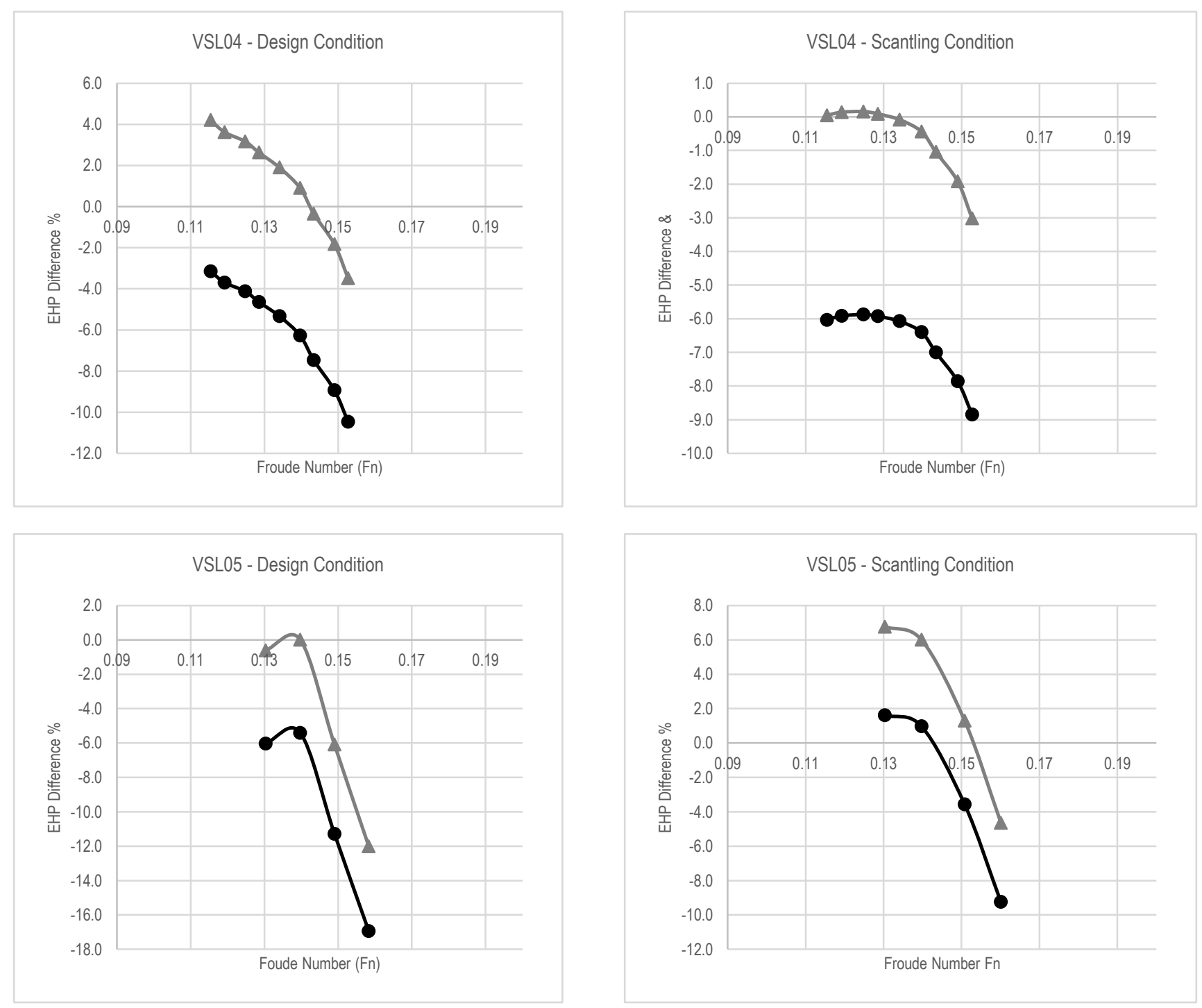

| 2
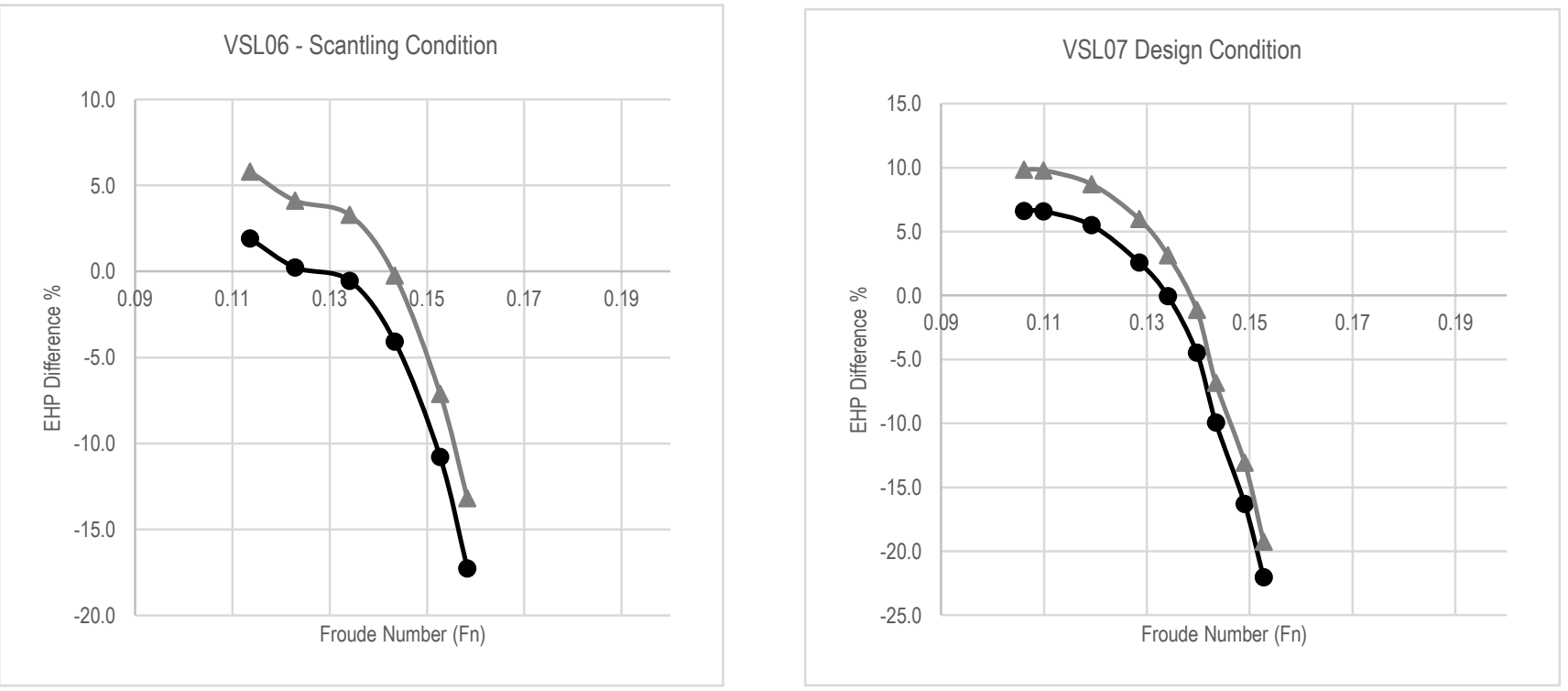

4 Figure [37] to [47]: EHP prediction error (\%) distribution over different speeds before and after Stage 1 calibration - LADEN 5 CONDITION 

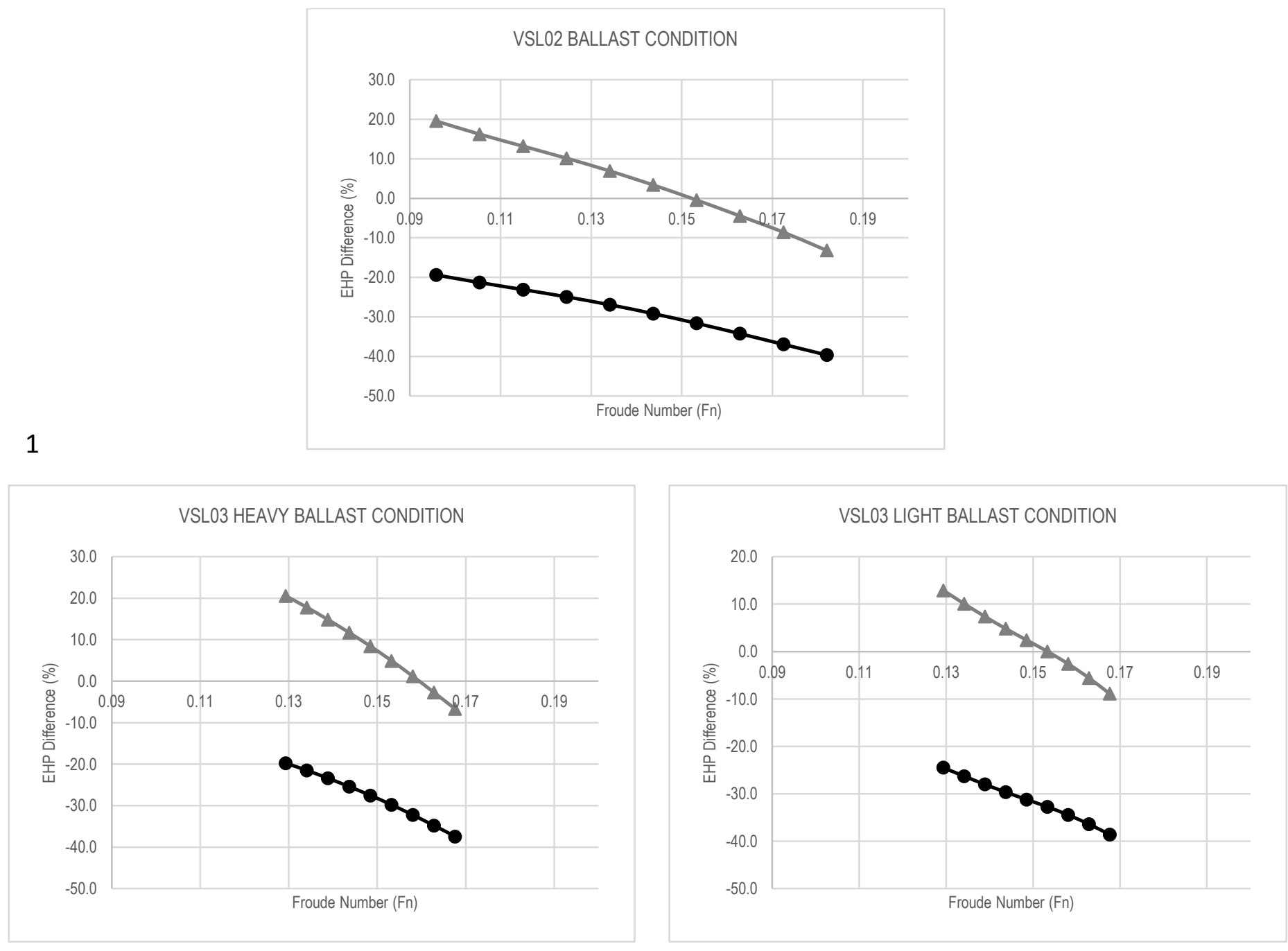

2
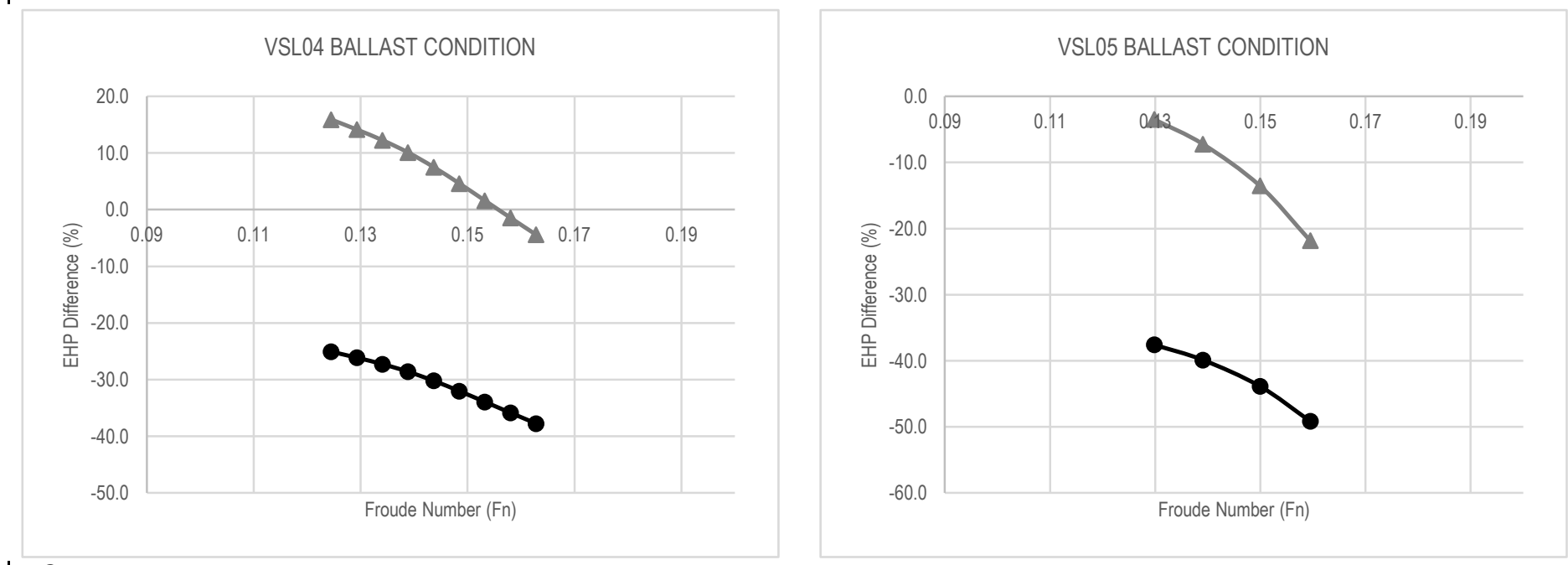

3

4

5

6 

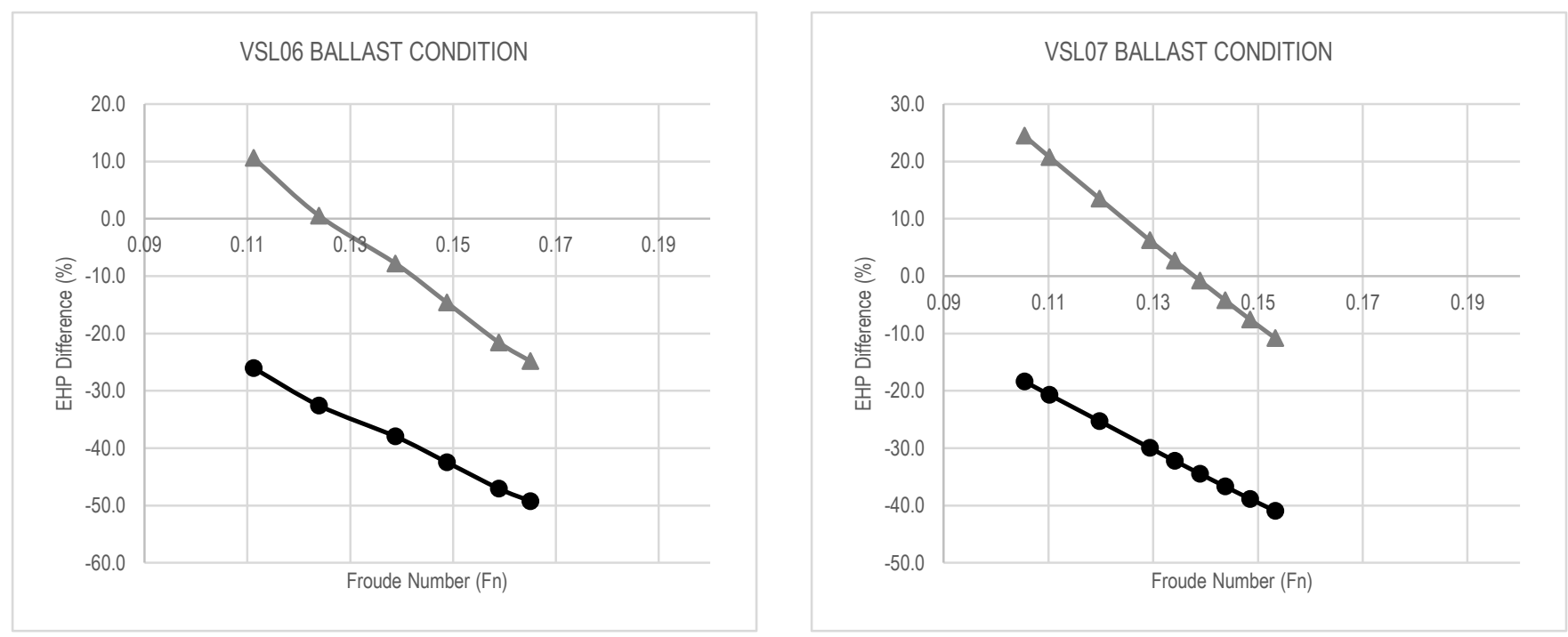

Figure [48] to [54]: EHP prediction error (\%) distribution over different speeds before and after Stage 1 calibration BALLAST CONDITION

\section{Interpretation of the Results}

When looking at the results for both Laden and Ballast conditions it is evident that the simulation had an obvious positive effect as in all vessel cases the prediction has been improved given the fact that the all EHP differences have been reduced, while all the trends in terms of deviation over speed have been accurately maintained. In fact, if one observes the form of the curves it is evident that the calibrated curves are in fact "translations" of the original publication curves towards the x-axis, which was after all the objective of the herein presented study.

\section{Laden Conditions:}

From Figures [37] to [47] the distribution of the EHP difference over difference speeds for the Laden Conditions is depicted with the following observations:

1. For 1 out of 11 cases (VSL03 - Design Condition) the translation of the deviation distribution was such that for the entire Froude number range there is an overestimation of the resistance which however is decreasing (on a $3^{\text {rd }}$ power basis) by increasing speed following the same trend as the equivalent curve prior to the calibration. The maximum overestimation is $-10 \%$ but by increasing Froude number it is reduced to almost $0.5 \%$.

2. Another interesting case would be that of VSL05 Design Condition, where for low speeds there is an underestimation which is decreasing by increasing curves starting from $1 \%$ at low Froude numbers and up to a minimum of $-2 \%$ overestimation and then sharply passes again to the underestimating region with the underestimation increasing sharply by increasing Froude numbers up to a maximum of $13 \%$.

3. For the 8 out of 11 cases the effect of the apparent translation of the deviation distribution curve is that there is an overestimation at low speeds of maximum $-10 \%$ which is decreased steeply by increasing speed up to a transition speed within the range of 0.125 to 0.175 Froude number from which point there is a transit to the underestimation area with a steep increase of underestimation by increasing speed up to a maximum $20 \%$ underestimation.

\section{Ballast Conditions:}

From Figures [48] to [54] the distribution of the EHP difference over difference speeds for the Ballast Conditions is depicted with the following observations:

1. For 1 out of 7 cases (VSL05), the deviation distribution is located at the underestimating region for all Froude numbers, starting from a minimum of $2 \%$ at low Froude numbers which is increasing on a power rate by increasing speed and up to a maximum of $20 \%$ at high Froude numbers.

2. For the rest 6 out of 7 cases, due to the translation of the error distribution curve an overestimation at low speeds is observed which is decreasing by increasing speed and at a transition speed of the region of 0.15 Froude number changes to the underestimation region which is increasing by increasing speed up to a maximum of $20 \%$.

3. For all cases and since the same trends of the curves prior to the calibration are kept the correlation between deviation and speed is very close to being linear and with a high steepness, which is on contrast to the Laden Condition which follows a $2^{\text {nd }-3 \text { rd }}$ power fit correlation.

From the above it can be deducted that the EHP calibration is successful and a very accurate prediction is possible for early 
Table [4] and Table [5] summarize the EHP difference between the Holtrop Prediction and the model test results prior and after the calibration for Laden and Ballast Conditions respectively. For the Laden conditions (both design and scantling) the improvement over all seems marginal as the deviation decreased from $5.7 \%$ to $4.7 \%$ in terms of absolute deviation. If one looks however to the nominal deviation the "translation" observed in the deviation graphs and described above, it has improved from an average underestimation of $1.4 \%$ to an overestimation of $-1.9 \%$ which is preferable in ship design studies in order to have a safer design margin for sizing the propulsion plant. For the laden conditions, one can also observe that despite an improvement of the prediction in some vessels of the herein presented study database is very distinct (VSL02, VSL03, VSL04, VSL05), for other vessels' in the same database and for the same selected optimization variant the prediction error is higher when compared to the respective error corresponding to the original Holtrop coefficients. This highlights a sensitivity of the method which is expected given the already low level of prediction error (\%) for the original Holtrop coefficients in the laden conditions.

In the Ballast conditions the average absolute deviation was reduced from $31.8 \%$ to $9.78 \%$ which makes it a considerable improvement that constitutes the basis for the next Stage 2 calibration for the Delivered Horse Power (SHP).

\begin{tabular}{|c|c|c|c|c|}
\hline \multicolumn{5}{|c|}{ STAGE 1 CALIBRATION RESULTS - LADEN CONDITIONS } \\
\hline Vessel & $\begin{array}{c}\text { Average } \\
\text { Error (\%) } \\
\text { EHP } \\
\text { After } \\
\text { Calibration }\end{array}$ & $\begin{array}{c}\text { Average } \\
\text { Absolute } \\
\text { Error (\%) } \\
\text { EHP } \\
\text { After } \\
\text { Calibration }\end{array}$ & $\begin{array}{c}\text { Average } \\
\text { Error (\%) } \\
\text { EHP } \\
\text { Original Holtrop } \\
\text { Coefficients }\end{array}$ & $\begin{array}{c}\text { Average } \\
\text { Absolute } \\
\text { Error (\%) } \\
\text { EHP } \\
\text { Original Holtrop } \\
\text { Coefficients }\end{array}$ \\
\hline VSL01 - Design Condition & -11.206 & 11.272 & -6.593 & 7.234 \\
\hline VSL02 - Scantling - Low Speed & -0.584 & 0.835 & 3.826 & 3.826 \\
\hline VSL02 - Design - Low Speed & -0.695 & 1.353 & 4.583 & 4.583 \\
\hline VSL03 - Design Condition & -7.035 & 7.035 & -3.620 & 4.063 \\
\hline VSL03 - Scantling Condition & -5.025 & 5.183 & -1.514 & 2.992 \\
\hline VSL04 - Design Condition & -1.194 & 2.454 & 6.008 & 6.008 \\
\hline VSL04 - Scantling Condition & 0.675 & 0.770 & 6.658 & 6.658 \\
\hline VSL05 - Design Condition & 4.668 & 4.677 & 9.908 & 9.908 \\
\hline VSL05 - Scantling Condition & -2.361 & 4.678 & 2.553 & 3.852 \\
\hline VSL06 - Scantling Condition & 1.222 & 5.627 & 5.088 & 5.807 \\
\hline VSL07 - Design Condition & 0.309 & 8.638 & 3.498 & 8.229 \\
\hline ENTIRE DATABASE & -1.930 & 4.775 & $\mathbf{2 . 7 6 3}$ & $\mathbf{5 . 7 4 2}$ \\
\hline
\end{tabular}

\begin{tabular}{|c|c|c|c|}
\hline \multicolumn{3}{|c|}{ STAGE 1 CALIBRATION RESULTS - BALLAST CONDITIONS } \\
\hline Vessel & $\begin{array}{c}\text { Average Error (\%) } \\
\text { EHP - NSGA11-885 }\end{array}$ & $\begin{array}{c}\text { Average Absolute Error } \\
\text { (\%) } \\
\text { EHP - NSGA11-885 }\end{array}$ & $\begin{array}{c}\text { Average Error (\%) } \\
\text { EHP - Original } \\
\text { Holtrop } \\
\text { Coefficients }\end{array}$ \\
\hline VSL02 - Ballast Condition & -4.269 & 9.604 & 28.725 \\
\hline VSL03 - Heavy Ballast Condition & -7.765 & 9.863 & 27.989 \\
\hline VSL03 -Light Ballast Condition & -2.298 & 6.086 & 31.310 \\
\hline VSL04 - Ballast Condition & -6.692 & 7.999 & 30.769 \\
\hline VSL05 - Ballast Condition & 11.517 & 11.517 & 37.813 \\
\hline VSL06 - Ballast Condition & 9.609 & 13.330 & 40.913 \\
\hline VSL07 - Ballast Condition & -4.933 & 10.119 & 30.823 \\
\hline ENTIRE DATABASE & -0.690 & 9.788 & 32.620 \\
\hline
\end{tabular}


25

\subsection{Stage 2: Shaft Power Calibration}

After the calibration of the constants that are parts of the equations that predict the bare hull resistance and in turn the effective power, Stage 2 of this calibration study includes the study of the self-propulsion problem, which can be reduced into the following equation [1]

$$
S H P=E H P * \frac{1-t}{1-w} * \eta_{R} * \eta_{0} * \eta_{s}
$$

Where:

1. $w$ is the wake fraction

2. $t$ is the thrust deduction

3. $\eta_{R}$ is the relative rotative efficiency

4. $\quad \eta_{0}$ is the propeller open water efficiency, as per model test results for each case

5. $\eta_{s}$ is the shafting system efficiency, as per model test results for each case

Since the EHP prediction is calibrated throughout Stage 1 runs, the calibration in Stage 2 focuses on the independent components of equation (1) in respective calibration runs through which the Shaft Power is calculated. It should be noted that at this point of the study it is important to have a clear decomposition and database with values for each component.

For the prediction of the wake fraction and the thrust deduction, in the herein proposed methodology the average of following empirical formulae (Table [6] and Table [7]) is considered. The reason for such an approach is that the Holtrop proposed formula exhibits a high instability and infidelity for primarily for the wake fraction coefficient. This according to the Authors' opinion can be attributed to the different stern geometries and viscous phenomena of the database selected versus the original database of Holtrop and Mennen.

\begin{tabular}{|l|c|}
\hline Schneekluth Formula & $w=0.5 * C_{P} * \frac{1.6}{1+\frac{D}{T} * \frac{16}{10+\frac{L}{B}}}$ \\
\hline Kruger Formula & $w=0.75 * C_{B}-0.24$ \\
\hline Heckscher Formula & $w=0.7 * C_{P}-0.18$ \\
\hline Troost Formula & $w=0.25+2.5 *\left(C_{B}-0.6\right)^{2}$ \\
\hline \multicolumn{2}{|c|}{ Table [6]: Formulae used for the prediction of the wake fraction in the self-propulsion problem }
\end{tabular}

27

\begin{tabular}{|l|c|}
\hline Holtrop and Mennen Formula & $t=0.001979 * \frac{L}{B *\left(1-C_{P}\right)}+1.0585 * \frac{B}{L}-0.00524-0.1418 * \frac{D^{2}}{B * T}$ \\
\hline Heckscher Formula & $t=0.5 * C_{P}-0.12$ \\
\hline Danckwardt & $t=0.5 * C_{B}-0.15$ \\
\hline
\end{tabular}

Table [7]: Formulae used for the prediction of the thrust deduction in the self-propulsion problem

29

In the approach proposed herein, the thrust deduction, relative rotative efficiency and wake fraction are estimated using the same database as in Stage 1. While in Stage 1 the solver used for the optimization problem (NSGAll) was of an evolutionary nature and on a multi-objective basis the problem, here a single-objective optimization problem is used and each of the coefficients $\left(t, w, \eta_{R}\right)$ is checked separately. The optimization target is the minimization of the average deviation of the wake fraction, thrust deduction and relative rotative efficiency respectively based on the available experimental data. For this the gradient-based method TSEARCH [12] algorithm is used which is also available in CAESES.

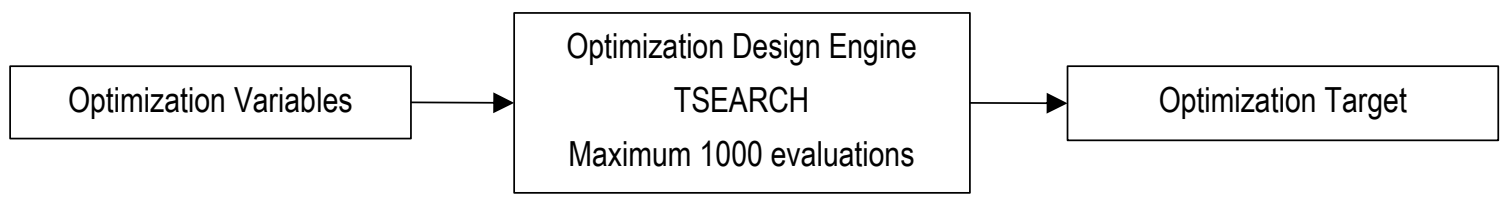

Figure [55]: The generic optimization problem in Stage 2

Based on the above approach and the range of parameters used as depicted in Tables [2] and [3] the results are presented below. 
2 The relative rotative efficiency prediction by the Holtrop and Mennen empirical formula illustrating a very impressive accuracy 3 for all vessel cases, both Laden and Ballast conditions and throughout the entire speed range when compared to the respective model test predictions. As one can see from Table [8] the deviation for the Laden condition ranged from -3.5\% overestimation to a $3 \%$ underestimation with the average being an overestimation of $-0.89 \%$, while for the Ballast condition the deviation ranged from a $-4.17 \%$ overestimation to a $3.75 \%$ underestimation with the average being a $-0.75 \%$ overestimation.

7 Given this very good accuracy it was not deemed necessary to proceed with any further calibration but rather to focus on the 8 wake fraction and thrust deduction coefficients calibration

10

11

12

13

14

15

16

17

18

19

20

21

22

23

24

25

26

27

28

29

\begin{tabular}{|c|r|r|}
\hline Case & EtaR Average Prediction Error (\%) & EtaR Average Absolute Prediction Error (\%) \\
\hline VSL02 - Scantling & -1.589 & 1.589 \\
\hline VSL02 - Design & -2.215 & 2.215 \\
\hline VSL03 - Design Condition & -2.209 & 2.209 \\
\hline VSL03 - Scantling Condition & -2.645 & 2.645 \\
\hline VSL04 - Design Condition & 1.630 & 1.630 \\
\hline VSL04 - Scantling Condition & 1.863 & 1.863 \\
\hline VSL05 - Design Condition & -0.334 & 0.407 \\
\hline VSL05 - Scantling Condition & -0.926 & 0.926 \\
\hline VSL06- Scantling Condition & 0.353 & 0.353 \\
\hline VSL07- Design Condition & -0.870 & 0.870 \\
\hline \multicolumn{2}{|c|}{} \\
\hline \multicolumn{2}{|c|}{ Table [8]: Deviation of the Relative Rotative Efficiency (\%) }
\end{tabular}

\section{Wake Fraction}

Given the sensitivity of the wake fraction on scale effects it should be noted that its prediction is challenging and cannot be accurately achieved with empirical methods. Also for the case of model test prediction the extrapolation and scaling to full scale wake results is something of not high fidelity, while the full scale CFD viscous calculation is a task requiring time and computational resources.

This is also reflected when we observe the deviation of the herein predicted wake fraction (averaged from 5 different formulae) when compared to the respective model test results (for full scale). The results of the single objective optimization problem can be seen on Table [9] for the Laden Conditions and Table [10] for the Ballast Conditions. It is evident that the prediction has been improved significantly from an average absolute deviation of $26.93 \%$ (overestimation) prior the calibration to a $3.08 \%$ (underestimation) equivalent after the calibration. For the Ballast Conditions the average reduced from $-6.51 \%$ to $2.08 \%$ underestimation after the calibration.

\section{Thrust Deduction}

Similar to the wake fraction the thrust deduction is also a coefficient highly dependent on the local phenomena and hence is very sensitive to each different hull form. The calibration converged also here yielding a significant improvement as shown on Table [9] for the Laden Conditions and Table [10] for the Ballast Conditions. The average error has been reduced rather drastically from $72.1 \%$ to $7.69 \%$ for the Laden Conditions and from $26.93 \%$ to $-3.08 \%$ for the Ballast Conditions

\begin{tabular}{|c|c|c|c|c|}
\hline Vessel & $\begin{array}{c}\text { T Average Error (\%) } \\
\text { After Stage 2 Calibration }\end{array}$ & $\begin{array}{c}\text { T Average } \\
\text { Error (\%) Prior } \\
\text { Calibration }\end{array}$ & $\begin{array}{c}\text { W Average Error (\%) } \\
\text { After Stage 2 Calibration }\end{array}$ & $\begin{array}{c}\text { W Average Error (\%) } \\
\text { Prior Calibration }\end{array}$ \\
\hline VSL02 - Scantling & -2.798 & 29.067 & -13.767 & 13.446 \\
\hline VSL02 - Design & -1.587 & 25.615 & -16.775 & 9.485 \\
\hline VSL03 - Design & -8.600 & 46.163 & 6.593 & 39.991 \\
\hline VSL03 - Scantling & -5.775 & 52.220 & 15.076 & 50.596 \\
\hline VSL04 - Design & -10.084 & 52.034 & 0.188 & 31.043 \\
\hline VSL04 - Scantling & 1.164 & 44.271 & 14.337 & 49.499 \\
\hline VSL05 - Design & 41.604 & 146.104 & -11.462 & 15.751 \\
\hline VSL05 - Scantling & 44.794 & 159.600 & -20.648 & 3.727 \\
\hline VSL06 - Scantling & 19.719 & 95.884 & -10.142 & 17.622 \\
\hline VSL07 - Design & -1.545 & 70.011 & 5.772 & 38.136 \\
\hline ENTIRE DATABASE & 7.688 & 72.097 & -3.082 & 26.930 \\
\hline
\end{tabular}




\begin{tabular}{|c|c|c|c|c|}
\hline Vessel & $\begin{array}{c}\text { T Average Error (\%) } \\
\text { After Stage } 2 \text { Calibration }\end{array}$ & $\begin{array}{c}\text { T Average Error } \\
(\%) \\
\text { Prior Calibration }\end{array}$ & $\begin{array}{c}\text { W Average Error (\%) } \\
\text { After Stage } 2 \text { Calibration }\end{array}$ & $\begin{array}{c}\text { W Average Error (\%) } \\
\text { Prior Calibration }\end{array}$ \\
\hline VSL02 - Ballast & -13.495 & -3.487 & -18.019 & -26.774 \\
\hline VSL03 - Heavy Ballast & -7.275 & 12.200 & 3.165 & -2.488 \\
\hline VSL03 -Light Ballast & -9.193 & 7.217 & -0.483 & -7.538 \\
\hline VSL04 - Ballast & 2.148 & 22.587 & 3.954 & 3.041 \\
\hline VSL05 - Ballast & 50.219 & 89.055 & -5.442 & -7.208 \\
\hline VSL06 - Ballast & 21.478 & 46.438 & -6.924 & -11.808 \\
\hline VSL07 - Ballast & -1.839 & 22.773 & 9.181 & 7.234 \\
\hline ENTIRE DATABASE & 6.0058 & 28.112 & -2.081 & -6.506 \\
\hline
\end{tabular}

1 Table [10]: Average Error (\%) for wake fraction and thrust deduction - BALLAST CONDITIONS

\section{Final SHP Prediction}

4 prediction. increasing speed.

\section{Laden Condition} following can be observed:

After each of the components of the self-propulsion equation are respectively calibrated as described above, one can assess their effect (combined with the results of the Stage 1/EHP calibration) on the shaft horsepower (SHP).

The effect is assessed herein both in terms of the prediction error at different speeds as well as the final speed power curves prior and after calibration for each vessel and loading condition.

For the error distribution, the translation phenomenon of the curves towards to lower percentages and overestimation areas observed in the EHP calibration is also evident here both in Laden and Ballast conditions. This result to overestimations of the required power in many cases (especially at low speed areas) leading to a more conservative approach of the power

Another interesting observation for both laden and ballast conditions is that the trend of SHP difference distribution of the original Holtrop coefficients is retained in most of the cases and is very similar (with some minor distortions) to the respective EHP distributions indicating the strong dependency of the SHP to EHP. This trend is increasing tend to underestimation by

From Figures [56] to [65] the SHP deviation distribution over different speeds is depicted for the Laden Conditions. The

1. For 5 out of 10 cases an overestimation of the SHP for the entire range of Froude numbers is observed from a maximum $20 \%$ at low Froude number range and up to $4 \%$ at the high range, indicating the same trend of increasing underestimation by increasing Froude number. The reason for the high overestimation at the low speeds in this case is the translation of the original curve to lower overestimating regions to attain an average close to zero deviation.

2. For 4 out of 10 cases while at the lower Froude number range there is an overestimation of up to $15 \%$ at higher Froude numbers that transcends to the underestimation region at a transition Froude number ranging from 0.125 to 0.175 depending on each vessel case, while the underestimation at the highest speeds is maximum $15 \%$.

3. An interesting case out of the above 5 mentioned, is VSL07 where up to 0.16 Froude number the overestimation is practically constant at $5 \%$ and then drops rapidly, transits to the underestimating region, increasing linearly and steeply by increasing speed with a maximum underestimation of $30 \%$.

4. For 1 out of 10 cases there is only underestimation for the all Froude numbers having the same trend as with the original case however translated to a much lower level of error. This can be seen from an underestimation of $1 \%$ at low Froude numbers and up to a maximum of $3 \%$ at higher. Interestingly for this case (VSL04 Scantling Condition) the difference from 12 to 14 knots is almost constant at $1 \%$ and changes rapidly from 0.135 to 0.15 Froude number with an almost linear increase by increasing speed. 

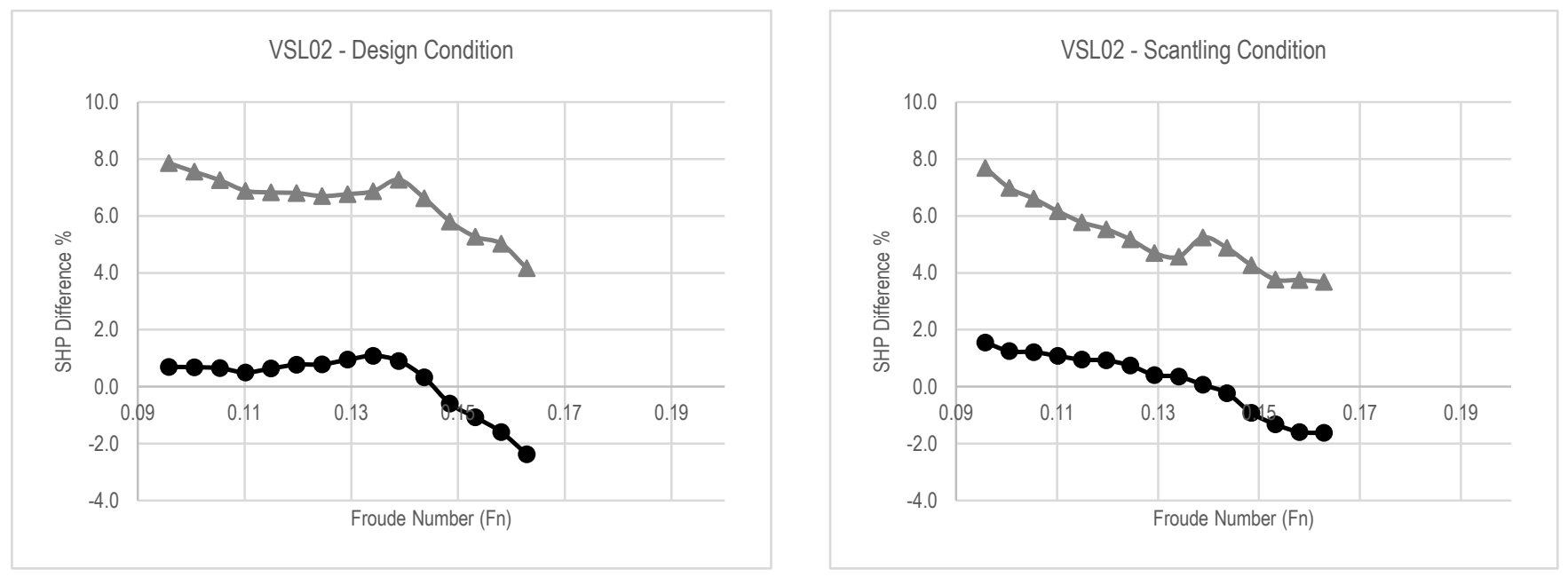

2
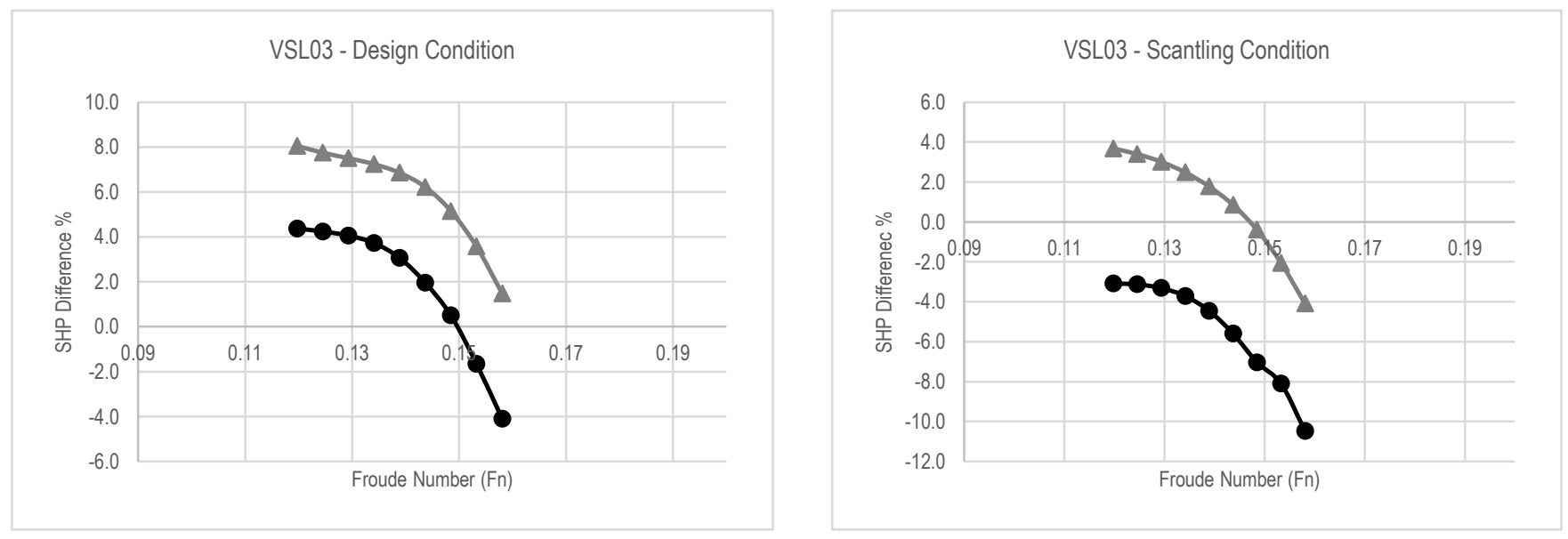

3
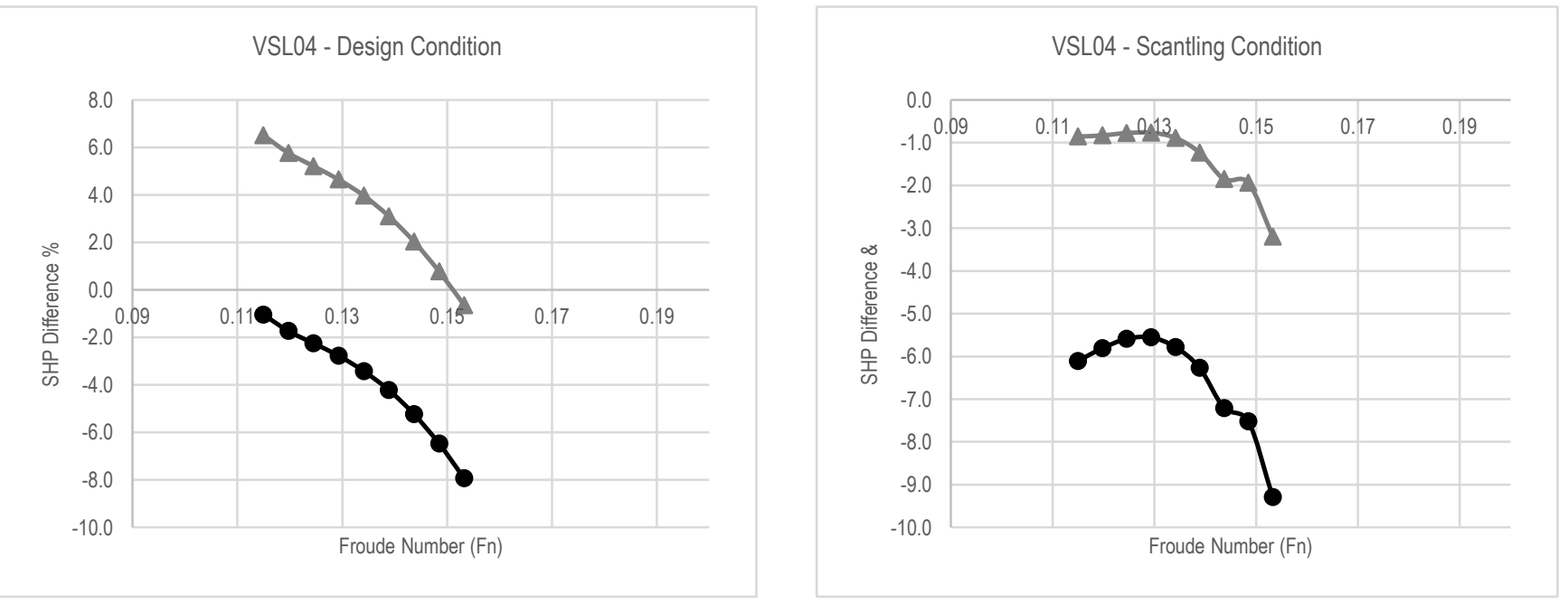

4
5
6
7 

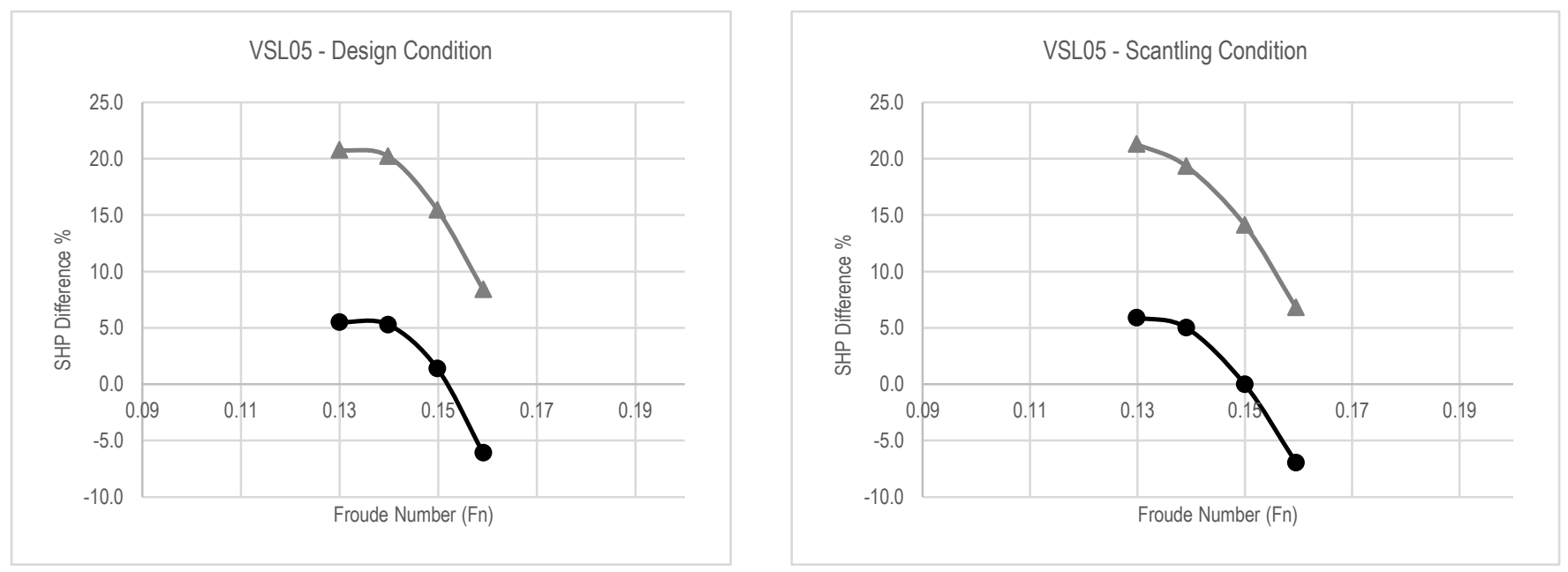

1
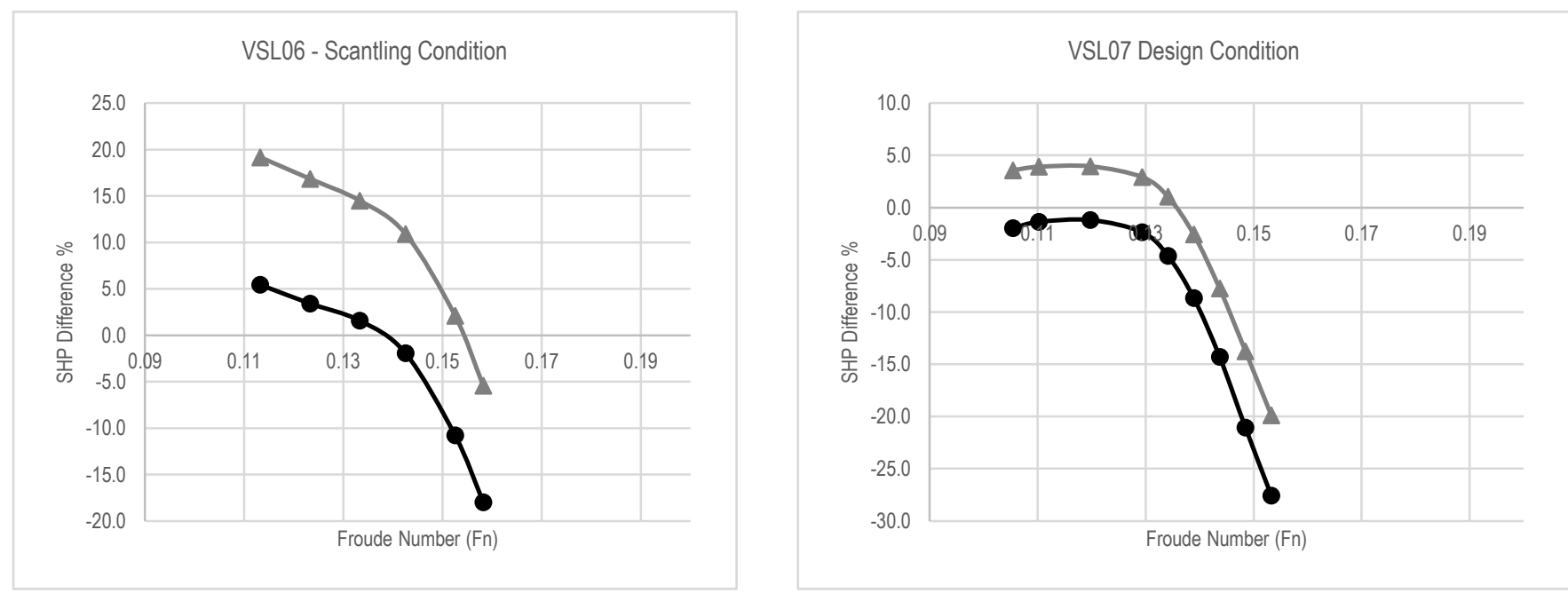

Figure [56] to [65]: SHP Prediction Error (\%) distribution over different speeds before and after Stage 2 calibration - LADEN CONDITION

\section{Ballast Condition}

From Figures [66] to [72] the SHP deviation distribution over different speeds is depicted for the Ballast Conditions. The following can be observed:

1. For 1 out of 7 cases (VSL05 ballast condition) the methodology underestimates the required power for the entire Froude number range with the latter descending by increasing speed and having the same trend as prior to the calibration.

2. For the rest 6 out of 7 cases there is an overestimation of the required power at low speeds of maximum $25 \%$ which by increasing speeds linearly decreases and at a Froude number at the region 0.15 (depending on each vessel) there is a transition to the underestimation region. The underestimation also increases linearly by increasing speed up to a maximum of $15 \%$. 

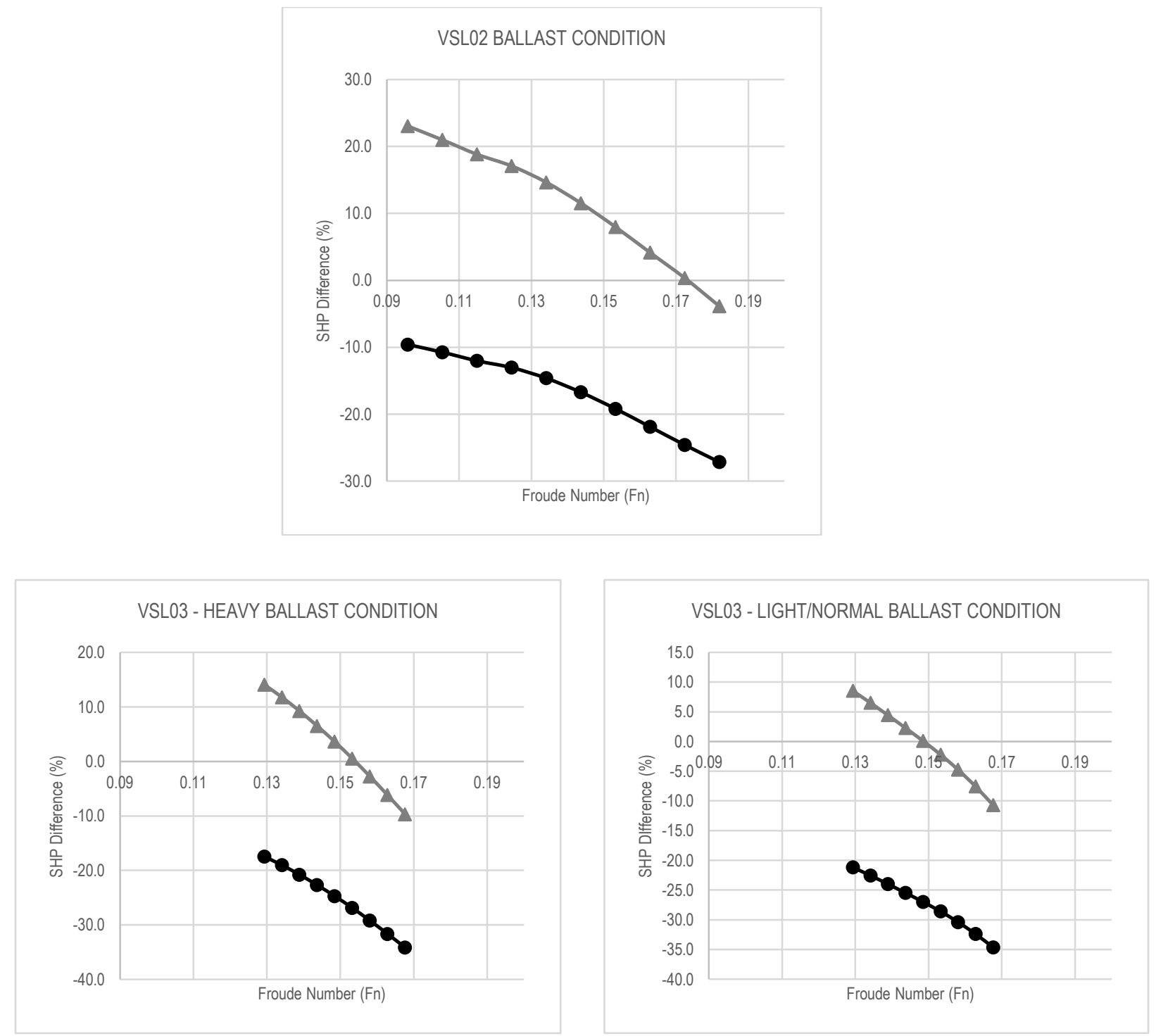

3
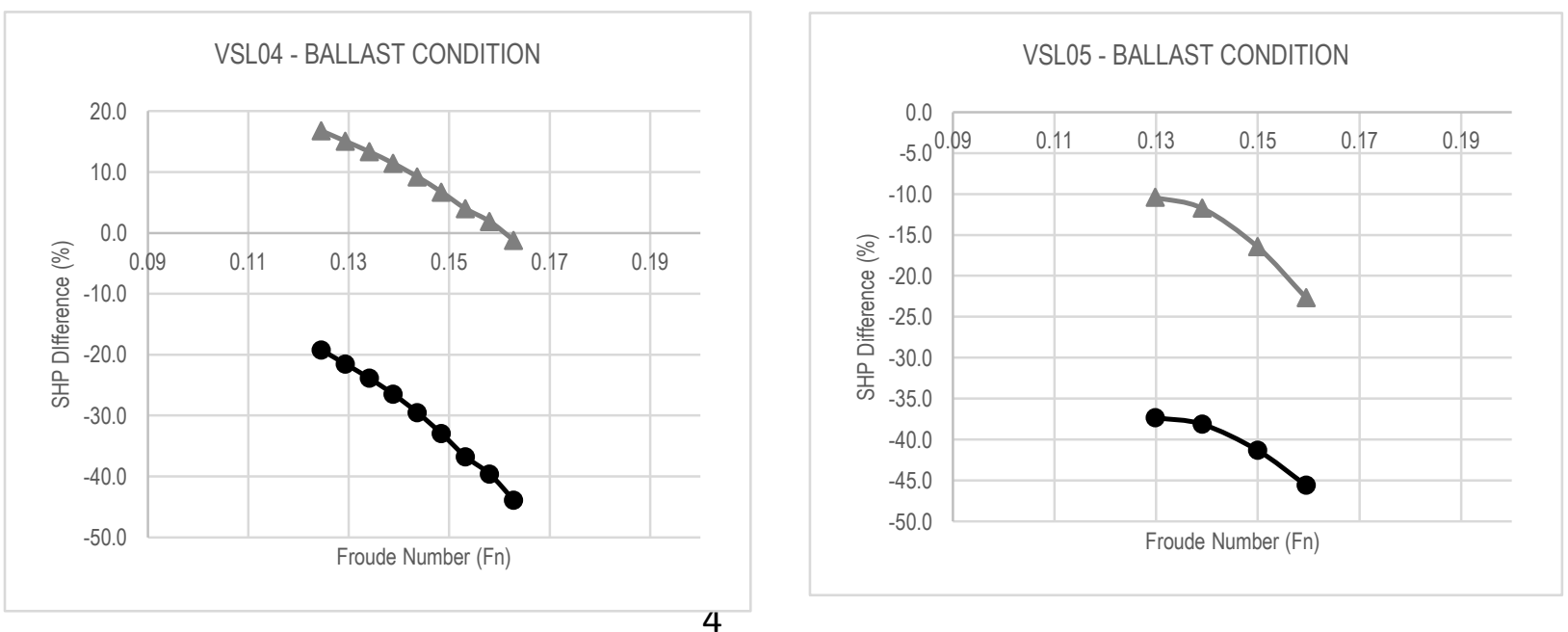

5

6 

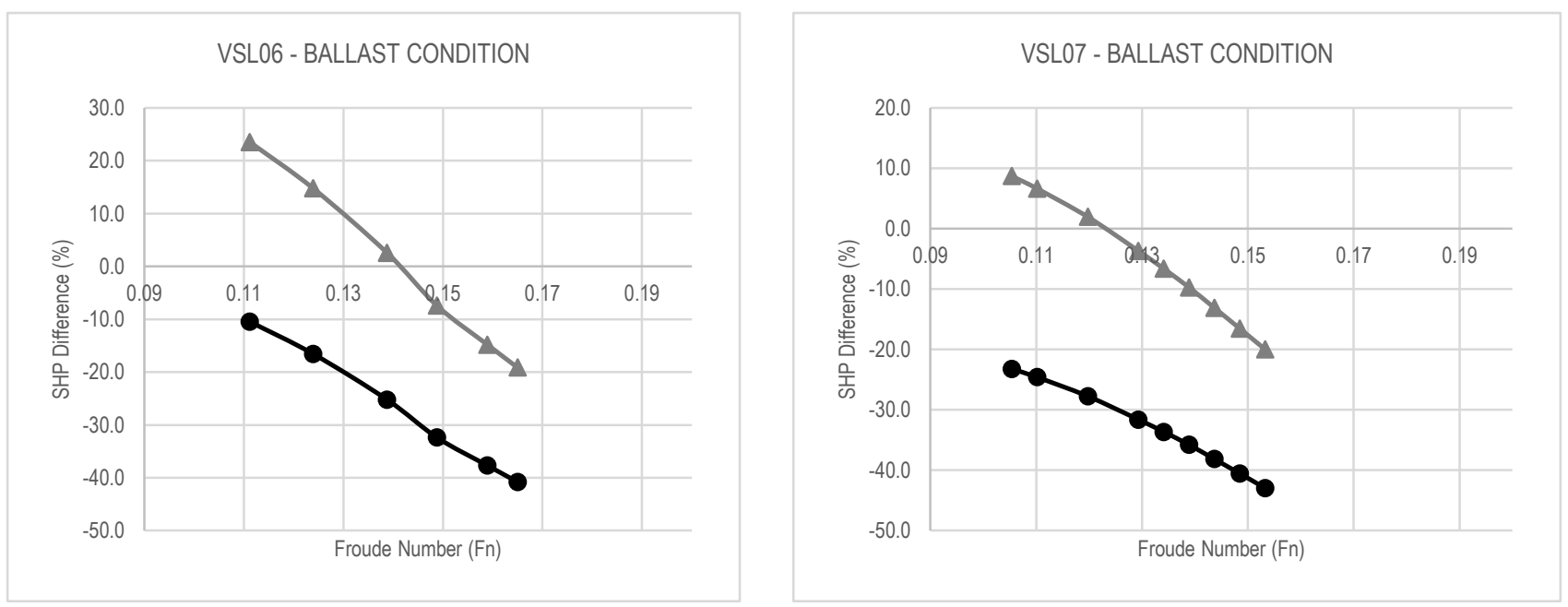

1

21

Figure [66] to [72]: SHP Prediction Error (\%) distribution over different speeds before and after Stage 2 calibration BALLAST CONDITION

In Table [11] (for the Laden conditions) and [12] (for the Ballast conditions) the deviation of the predicted required Delivered Horsepower (SHP) prior the calibration (original Holtrop coefficients) and after the calibration, when compared to the model test prediction is depicted. The average absolute error (\%) has been significantly reduced from $28.68 \%$ to $10.3 \%$ for the Ballast Conditions. For the Laden Conditions however, the average absolute error (\%)) increased marginally from $4.59 \%$ to $7.49 \%$. The reason for this is the already small prediction error. By trying to improve such a small error the sensitivity is at a level that can improve the prediction accuracy for some cases and deteriorate for others. A typical example is VSL02 for which the prediction error decree increased from $0.95 \%$ to $5 \%$ while in the meantime and same combination of variables the prediction error of VSL02, for VSL04 decreased from $6.57 \%$ to $1.37 \%$. It is therefore advised from Authors not to attempt such calibration studies in applications where the prediction error is already very small due to inherent sensitivity and volatility issues of the method.

In addition to the above it should also be noted, that when looking at the average deviation (not absolute) this has been an average underestimation of $1.74 \%$ prior to the calibration which has been changed to an average overestimation of $-4.65 \%$ which leads to a safer margin for predictions at the preliminary ship design stages.

Lastly, all the individual components have an improved accuracy, thus the herein proposed calibrated methodology depicts in a more accurate way the sensitivities of the methodology in main dimensions and design characteristics for all the resistance sub-components which is very useful for preliminary ship design studies.

\begin{tabular}{|c|c|c|c|c|}
\hline Vessel & $\begin{array}{l}\text { SHP Average Error (\%) } \\
\text { After Stage } 2 \text { Calibration }\end{array}$ & $\begin{array}{c}\text { SHP Average Absolute } \\
\text { Error (\%) } \\
\text { After Stage } 2 \text { Calibration }\end{array}$ & $\begin{array}{l}\text { SHP Average Error (\%) } \\
\text { Prior Stage } 2 \text { Calibration }\end{array}$ & $\begin{array}{c}\text { SHP Average Absolute } \\
\text { Error (\%) } \\
\text { Prior Stage } 2 \text { Calibration }\end{array}$ \\
\hline VSL02 - Scantling Condition & 5.250 & 5.250 & 0.188 & 0.948 \\
\hline VSL03 - Design Condition & 5.977 & 5.977 & 1.793 & 3.073 \\
\hline VSL03 - Scantling Condition & 0.959 & 2.4151 & -5.434 & 5.434 \\
\hline VSL04 - Design Condition & 3.482 & 3.625 & -3.898 & 3.898 \\
\hline VSL05 - Scantling Condition & 15.408 & 15.408 & 1.008 & 4.472 \\
\hline VSL06 - Scantling Condition & 9.682 & 11.496 & -3.375 & 6.855 \\
\hline VSL07 - Design Condition & -3.170 & 6.594 & -9.227 & 9.227 \\
\hline ENTIRE DATABASE & 5.894 & 7.487 & -2.382 & 4.596 \\
\hline
\end{tabular}




\begin{tabular}{|c|c|c|c|c|}
\hline Vessel & $\begin{array}{c}\text { SHP Average Error } \\
(\%) \\
\text { After Stage } 2 \\
\text { Calibration }\end{array}$ & $\begin{array}{l}\text { SHP Average Absolute Error } \\
\qquad(\%) \\
\text { After Stage } 2 \text { Calibration }\end{array}$ & $\begin{array}{c}\text { SHP Average Error } \\
(\%) \\
\text { Prior Stage } 2 \\
\text { Calibration }\end{array}$ & $\begin{array}{c}\text { SHP Average Absolute Error } \\
(\%) \\
\text { Prior Stage } 2 \text { Calibration }\end{array}$ \\
\hline VSL02 - Ballast - Low Speed & 11.467 & 12.240 & -16.954 & 16.954 \\
\hline VSL03 - Heavy Ballast Condition & 3.0225 & 7.154 & -25.178 & 25.178 \\
\hline VSL03 -Light Ballast Condition & -0.3793 & 5.228 & -27.354 & 27.354 \\
\hline VSL04 - Ballast Condition & 8.577 & 8.843 & -30.424 & 30.424 \\
\hline VSL05 - Ballast Condition & -15.331 & 15.331 & -40.589 & 40.589 \\
\hline VSL07 - Ballast Condition & -5.820 & 9.668 & -33.141 & 33.141 \\
\hline ENTIRE DATABASE & 0.207 & 10.311 & -28.689 & 28.689 \\
\hline
\end{tabular}

Table [12]: Average Deviation of the Delivered Horse Power (\%) per vessel - BALLAST Condition

3 The below table [13] summarizes, the finalized values chosen for the Holtrop constants in terms of resistance and propulsion

4 power prediction formula following the two stage optimization and calibration process.

5

\begin{tabular}{|c|c|c|c|c|c|}
\hline No. & $\begin{array}{r}\text { Value in Original } \\
\text { Holtrop Publication } \\
\text { LADEN }\end{array}$ & $\begin{array}{r}\text { Value After Final } \\
\text { Calibration } \\
\text { LADEN }\end{array}$ & No. & $\begin{array}{r}\text { Value in Original Holtrop } \\
\text { Publication } \\
\text { BALLAST }\end{array}$ & $\begin{array}{r}\text { Value After Final Calibration } \\
\text { BALLAST }\end{array}$ \\
\hline $\mathrm{L}-1$ & 2223105 & 2242064.546 & B-1 & 2223105 & 2207306.02 \\
\hline $\mathrm{L}-2$ & 3.78613 & 2.965053788 & B-2 & 3.78613 & 2.369306477 \\
\hline $\mathrm{L}-3$ & 1.07961 & 0.987251087 & B-3 & 1.07961 & 0.965964752 \\
\hline $\mathrm{L}-4$ & -1.37565 & -1.248029297 & B-4 & -1.37565 & -1.426852827 \\
\hline $\mathrm{L}-5$ & -1.89 & -1.585484092 & B-5 & -1.89 & -1.865680934 \\
\hline L-6 & 0.56 & 0.888603037 & B-6 & 0.56 & 0.310946822 \\
\hline L-7 & 0.31 & 0.679055924 & B-7 & 0.31 & 0.599398947 \\
\hline $\mathrm{L}-8$ & 1.5 & 0.679055924 & B-8 & 1.5 & 2.660425727 \\
\hline $\mathrm{L}-9$ & 0.8 & 0.311482414 & B-9 & 0.8 & 0.866790265 \\
\hline$L-10$ & -1.69835 & -1.968221561 & B-10 & -1.69835 & -1.492373541 \\
\hline $\mathrm{L}-11$ & $c_{15_{\text {new }}}=0$ & 1.215701534 & B-11 & $c_{15_{\text {new }}}=0$ & -0.311146715 \\
\hline $\mathrm{L}-12$ & 0.0140407 & 0.022718547 & $\mathrm{~B}-12$ & 0.0140407 & 0.01282916 \\
\hline$L-13$ & 1.75254 & 1.420567636 & B-13 & 1.75254 & 1.28395819 \\
\hline L-14 & 4.79323 & 5.681261921 & B-14 & 4.79323 & 4.854474708 \\
\hline L-15 & 2 & 2.580880446 & B-15 & 2 & 2.202572671 \\
\hline L-16 & -0.1 & -0.147266651 & B-16 & -0.1 & -0.405693141 \\
\hline $\mathrm{L}-17$ & -2 & -2.988265812 & B-17 & -2 & 1.654390784 \\
\hline L-18 & 1.73014 & 1.465613794 & $\mathrm{~B}-18$ & 8.07981 & 7.892027161 \\
\hline L-19 & 0.7067 & 0.6426 & B-19 & -13.8673 & -12.1206 \\
\hline$L-20$ & $c_{16 C_{n e w}}=0$ & 1.516899 & B-20 & 6.984388 & 9.3225 \\
\hline $\mathrm{L}-21$ & $c_{16 D_{\text {new }}}=0$ & -0.340063 & $\mathrm{~B}-21$ & 1 & 1.3920 \\
\hline $\mathrm{L}-22$ & 1.446 & 1.815469596 & B-22 & 2 & 2.8830 \\
\hline$L-23$ & 0.03 & 0.023458457 & B-23 & 3 & 3.1013 \\
\hline L-24 & 0 & 0.318760967 & B-24 & 1.446 & 2.238521401 \\
\hline $\mathrm{L}-25$ & -0.9 & -1.202760357 & B-25 & 0.03 & 0.4431 \\
\hline $\mathrm{L}-26$ & -2 & -2.374135958 & B-26 & 0 & 0.0587 \\
\hline L-27 & 0.2228446 & 0.377695125 & B-27 & -0.9 & -0.3324 \\
\hline L-28 & 0.92497 & 0.668810559 & B-28 & -2 & -1.8767 \\
\hline $\mathrm{L}-29$ & -0.521448 & -0.623591974 & B-29 & 48.2 & 28.87945373 \\
\hline L-30 & 0.6906 & 0.766962692 & B-30 & 2.078 & 3.323125048 \\
\hline $\mathrm{L}-31$ & 0 & -2.825314717 & B-31 & 0.479948 & 0.446070497 \\
\hline $\mathrm{L}-32$ & 0.56 & 0.843195239 & B-32 & 0.92497 & 0.613664454 \\
\hline L-33 & 0.11 & 0.095151446 & B-33 & -0.521448 & -0.565916533 \\
\hline L-34 & -3 & -3.153574426 & B-34 & 0.6906 & 0.532242313 \\
\hline L-35 & 2 & 3.251325246 & B-35 & 0.003 & 0.01538851 \\
\hline L-36 & 3 & 2.339742123 & B-36 & 0 & 1.952800793 \\
\hline L-37 & 1.5 & 1.458429847 & B-37 & 0.56 & 0.354276341 \\
\hline L-38 & 2 & 2.337720302 & B-38 & 0.11 & 0.067300832 \\
\hline L-39 & 2 & 3.939871824 & B-39 & -3 & -3.752483406 \\
\hline $\mathrm{L}-40$ & 0.2 & 0.223463188 & B-40 & 2 & -1.800297551 \\
\hline L-41 & 0.006 & 0.069377752 & B-41 & 3 & 3.084248112 \\
\hline L-42 & -0.16 & -0.417208057 & B-42 & 1.5 & 1.358590066 \\
\hline$L-43$ & -0.00205 & -0.034246056 & B-43 & 2 & 1.663599603 \\
\hline
\end{tabular}




\begin{tabular}{|c|c|c|c|c|c|}
\hline L-44 & 0.003 & 0.125382971 & B-44 & 2 & 1.456360723 \\
\hline L-45 & 7.5 & 3.736263066 & B-45 & 0.2 & 0.885375296 \\
\hline L-46 & 4 & 4.217013809 & B-46 & 0.006 & 0.223290471 \\
\hline L-47 & & 0.036289769 & B-47 & -0.16 & -0.092530251 \\
\hline L-48 & $c_{\text {Anew }}=0$ & 2.948729686 & B-48 & -0.00205 & 0.082350805 \\
\hline L-49 & $c_{\text {Bnew }}=0$ & 0.013092241 & B-49 & 0.003 & 0.095168963 \\
\hline L-50 & 0.9922 & 0.9922 & B-50 & 7.5 & 6.146944381 \\
\hline L-51 & 0.05908 & 0.05908 & B-51 & 0.04 & 0.478261311 \\
\hline L-52 & 0.07424 & 0.07424 & B-52 & $c_{\text {Anew }}=0$ & 0.36520943 \\
\hline L-53 & 0.0225 & 0.0225 & B-53 & $c_{\text {Bnew }}=0$ & -0.673182269 \\
\hline L-54 & 0.001979 & 0.0001 & B-54 & $c_{\text {4new }}=1$ & 3.186513619 \\
\hline L-55 & 1.0585 & 3 & B-53 & 0.9922 & 0.9922 \\
\hline L-56 & -0.00524 & -0.107307217 & B-54 & 0.05908 & 0.05908 \\
\hline L-57 & -0.1418 & -0.001 & B-55 & 0.07424 & 0.07424 \\
\hline L-58 & 0.0661875 & 0.0661875 & B-56 & 0.0225 & 0.0225 \\
\hline L-59 & 1.21756 & 1.21756 & B-57 & 0.001979 & 0.00001 \\
\hline L-60 & 0.24558 & 0.24558 & B-58 & 1.0585 & 1.403225943 \\
\hline L-61 & 0.09726 & 0.09726 & B-59 & -0.00524 & -0.032286653 \\
\hline L-62 & 0.11434 & 0.11434 & B-60 & -0.1418 & -0.01 \\
\hline L-63 & 0.08333333 & 0.08333333 & B-61 & 0.0661875 & 0.0661875 \\
\hline L-64 & 1.3333 & 1.3333 & B-62 & 1.21756 & 1.21756 \\
\hline L-65 & 0.5 & 0.432109375 & B-63 & 0.24558 & 0.24558 \\
\hline L-66 & 1.6 & 1.325859375 & B-64 & 0.09726 & 0.09726 \\
\hline L-67 & 16 & 15.72532144 & B-65 & 0.11434 & 0.11434 \\
\hline L-68 & 0.75 & 0.662024983 & B-66 & 0.08333333 & 0.08333333 \\
\hline L-69 & 0.24 & 0.176972156 & B-67 & 1.3333 & 1.3333 \\
\hline L-70 & 0.7 & 0.316622043 & B-68 & 0.5 & 0.5 \\
\hline L-71 & -0.18 & -0.101944784 & B-69 & 1.6 & 1.6 \\
\hline L-72 & 0.25 & 0.25 & B-70 & 16 & 16 \\
\hline \multirow[t]{6}{*}{ L-73 } & 2.5 & 2.5 & B-71 & 0.75 & 0.820510639 \\
\hline & & & B-72 & 0.24 & 0.102842033 \\
\hline & & & B-73 & 0.7 & 0.431767617 \\
\hline & & & B-74 & -0.18 & -0.048268475 \\
\hline & & & B-75 & 0.25 & 0.252269833 \\
\hline & & & B-76 & 2.5 & 0.1 \\
\hline
\end{tabular}

\section{Uncertainty Analysis for Ship Design Applications}

Following the systematic calibration and for a given, final prediction error (\%) per vessel per speed, the latter was examined in order to be modelled for corrections in new prediction applications. The error was decided to be modeled by means of a non-linear regression formula generated in the IBM SPSS Software, for the database used herein for calibration and for the coefficients deriving from the Stage 2 calibration. This has been modelled in the below equations (2) and (3).

$\operatorname{Error}(\%)_{L A D E N}=0.008 * L_{B P}{ }^{-8.096}+0.009 * B^{-16.346}+0.006 * T_{m}{ }^{-34.137}+0.039 * C_{B}{ }^{1100.918}-0.014 * W S^{-4.877}$ (2)

(3)

$\begin{aligned} \operatorname{Error}(\%)_{B A L L A S T} & =-1132.36 * L_{B P}{ }^{-0.055}-95.054 * B^{-0.275}+911.906 * T_{m}{ }^{-0.080}+158.633 * C_{B}^{2.854}+0.256 \\ & * W S^{0.457}\end{aligned}$

$$
* W S^{0.457}
$$

$L_{B P}$ :

Length between perpendiculars

$B: \quad$ Breadth (moulded)

$T_{m}$ : $\quad$ Midship Draft

$C_{B}$ : $\quad$ Block Coefficient

WS: Wetted Surface

\section{Summary, Conclusions and Next Steps}

The methodology presented herein has provided an extensive calibration of the constants and coefficients of the Holtrop and Mennen Empirical Power Prediction Method based on a database of model test results of modern commercial vessels, as well as useful analysis on its accuracy over different speeds and hull forms but only focusing on full hull forms of a low Froude number. In general the accuracy of the original method can be considered adequate however decreasing constantly while the 
Froude number increases. After the utilization of the herein developed, multi-staged optimization approach for calibrating the methodologies the EHP prediction has been improved from $5.7 \%$ to $4.7 \%$ for the laden and from $32.6 \%$ to $9.7 \%$ for the ballast condition. The SHP prediction through the self-propulsion equation utilized the same model test database used in the EHP calibration. Finally, the accuracy of the SHP is improved from an error of $28.7 \%$ to $10.3 \%$ for the ballast condition while for the laden conditions the error is marginally increased from $4.59 \%$ to $7.49 \%$ despite the $1 \%$ improvement during the EHP calibration stage. This slight deterioration is attributed to the sensitivity of the method and the already very high level of accuracy (error level of about $5 \%$ is very small for an empirical method).

The uncertainty of the original as well as reproduced methodology has been examined and statistically modeled by a nonlinear regression analysis, for future use in Power prediction during the preliminary stages of various Ship Design studies. Next steps in this study will be the verification with CFD of the final results as well as the calibration based on databases generated from automated hull variation and subsequent CFD calculation.

What the reader should bear in mind as a conclusion is that the study presented herein doesn't propose an updated view of the established Holtrop and Mennen methodology rather than a rationale of calibrating and adapting this method to specific applications depending on the available data from the user. When having this mentality in mind during ship design the Naval Architect can adapt the resistance prediction methodology on parent and similar vessels and achieve a higher level of accuracy in the early stages.

\section{References}

1. Specialist Committee on CFD in Marine Hydrodynamics - Final Report and Recommendations to the $27^{\text {th }}$ ITTC, Copenhagen 2014.

2. Schneekluth, H. and Bertram, V. (1998), "Ship Design for Efficiency and Economy", Second edition, ButterworthHeinemann, ISBN 0750641339.

3. Holtrop, J.A, Mennen G.G.J.,(1982), "An approximate power prediction method", International Shipbuilding Progress 29.

4. Holtrop, J.A, (1984), "Statistical reanalysis of resistance and propulsion data", International Shipbuilding Progress 31

5. MARIN Report, "Holtrop-Mennen founders reveal the secret of method's long-lasting success", August 2010, Volume 100

6. Lu R., Turan O., Boulougouris E., Banks C., Incecik A. (2015), "A semi-empirical ship operational performance prediction model for voyage optimization towards energy efficient shipping", Journal of Ocean Engineering 110 (2015).

7. Mao W., Rychlik I., Wallin J., Storhaug G., (2016), "Statistical models for the speed prediction of a container ship", Journal of Ocean Engineering 126 (2016).

8. Vettor R., Guedes Soares C., (2016),"Development of a ship weather routing system", Journal of Ocean Engineering 123 (2016).

9. Cichowicz J., Theotokatos G., Vassalos D., (2015), "Dynamic Energy Modelling for ship life-cycle performance assessment", Journal of Ocean Engineering 110 (2015).

10. Deb K., Pratap A., Agarwaj S., Meyarivan T. (2002), "A fast and elitist multiobjective genetic algorithm: NSGA-II", IEEE Transaction on Evolutionary Computation, Volume 6, Issue 2, April 2002.

11. Ulungu E.L, Teghem J., Fortemps P.H, Tuyttends D. (1999), "MOSA method: a tool for solving multiobjective combinatorial optimization problems" Journal of Multi-Criteria Decision Analysis, Volume 8, Issue 4, July 1999.

12. Hilleary, R.R. The Tangent Search Method of Constrained Minimization; U.S. Naval Postgraduate School: Monterey, CA, USA, 1966. 